Review article

\title{
Immune cells as tumor drug delivery vehicles
}

\author{
Francis Combes $^{\mathrm{a}, \mathrm{b}}$, Evelyne Meyer ${ }^{\mathrm{b}, \mathrm{c}}$, Niek N. Sanders ${ }^{\mathrm{a}, \mathrm{b}, *}$ \\ ${ }^{a}$ Laboratory of Gene Therapy, Department of Nutrition, Genetics and Ethology, Faculty of Veterinary Medicine, Ghent University, Heidestraat 19, 9820 Merelbeke, Belgium \\ ${ }^{\mathrm{b}}$ Cancer Research Institute Ghent (CRIG), 9000 Ghent, Belgium \\ ${ }^{\mathrm{c}}$ Department of Pharmacology, Toxicology and Biochemistry, Faculty of Veterinary Medicine, Ghent University, Salisburylaan 133, 9820 Merelbeke, Belgium
}

\section{A R T I C L E I N F O}

\section{Keywords:}

Targeted drug delivery

Immune cells

Tumor microenvironment

Circulatory half-life

Conditional release

\begin{abstract}
A B S T R A C T
This review article describes the use of immune cells as potential candidates to deliver anti-cancer drugs deep within the tumor microenvironment. First, the rationale of using drug carriers to target tumors and potentially decrease drug-related side effects is discussed. We further explain some of the current limitations when using nanoparticles for this purpose. Next, a comprehensive step-by-step description of the migration cascade of immune cells is provided as well as arguments on why immune cells can be used to address some of the limitations associated with nanoparticle-mediated drug delivery. We then describe the benefits and drawbacks of using red blood cells, platelets, granulocytes, monocytes, macrophages, myeloid-derived suppressor cells, T cells and NK cells for tumor-targeted drug delivery. An additional section discusses the versatility of nanoparticles to load anti-cancer drugs into immune cells. Lastly, we propose increasing the circulatory half-life and development of conditional release strategies as the two main future pillars to improve the efficacy of immune cell-mediated drug delivery to tumors.
\end{abstract}

\section{Off-target side effects limit drug efficacy}

The current view of cancer is clearly that of a systemic disease [1]. Consequently, the containment of a cancerous mass or a tumor relies on its interaction with non-cancerous cells of the body. Back-and-forth communication between cancer cells and the cells in close proximity establishes a tumor microenvironment (TME) which consists of the extracellular matrix (ECM), fibroblasts, blood vessels and immune cells [2]. Ultimately, the co-evolution and dynamic interaction of these TME components will dictate the clinical progression of cancer.

Due to its heterogeneous nature, treatment of solid tumors requires a multimodal approach where surgery and radiation therapy has to be succeeded by adjuvant chemotherapy [3]. In search for decreasing the sometimes severe side effects due to off-target action on healthy cells, researchers in the 1980's experimented with drug delivery vehicles such as liposomes loaded with doxorubicin [4]. This coincided with the large scale manufacturing of monoclonal antibodies [5] which opened the gates to the identification of many additional therapeutic targets and was the onset of tumor targeted drug delivery [6]. Mapping of the human genome in the 1990s [7] facilitated the in-depth understanding of the genetic basis of cancer and revealed many novel therapeutic targets associated with this disease [8]. The increased knowledge on preventing, diagnosing and treating cancer is reflected in the substantially improved clinical outcome compared to that of half a century ago (WHO-IARC). Nevertheless, there is still a formidable task lying ahead since the majority of cancer-related mortality is currently associated with metastatic disease [9-11], a far more elusive target than the primary tumor.

When administrating drugs in vivo, the pharmacokinetics (i.e. the mechanisms governing the absorption, distribution, metabolism and excretion) of these compounds has to be carefully considered [12,13]. Typically, limited bioavailability and activity of therapeutics are a direct result of their inappropriate physicochemical properties and their fast clearance by e.g. enzymatic degradation, glomerular filtration and phagocytosis by cells of e.g. the mononuclear phagocyte system (MPS) [14]. Employing higher dosages, other administration routes or more frequent administrations can increase the drug plasma concentration, but also elicits more side effects. A common practice to treat cancer is administrating the maximum tolerated dosing (MTD) of the chemotherapeutic [15], which is the highest dose that does not cause unacceptable side effects within a specified time period [14,16-18]. However, drug dosages that are below complete efficacy (even at MTD) cause selective pressure on the cancer cells when repeatedly used. By evolutionary principle, sensitive cancer cells are weeded out while resistant cancer cells remain intact, proliferate and compose a new tumor resistant to the used therapeutic [18]. The genetic heterogeneity of

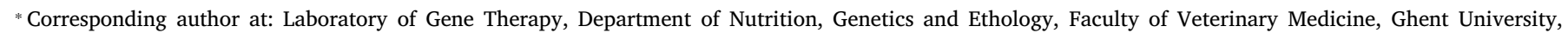
Heidestraat 19, 9820 Merelbeke, Belgium.

E-mail address: Niek.Sanders@ugent.be (N.N. Sanders). 
cancer cells is even more pronounced when they spread across multiple organs [11]. Employing combination therapies or frequently switching to other drugs helps addressing this problem [19], but oncologists are always limited by the MTD since off-target effects on healthy cells cause side effects and thus diminish the dose at which the therapeutic agent can be used. Moreover, as most anti-cancer drugs are administered systemically, they come into contact with numerous healthy cells.

\section{The tumor microenvironment as a more reliable therapeutic target}

Compared to the ever-changing cancer cells themselves, more stable tumor properties can be found in the TME of both primary tumors and metastatic niches [20-22]. For example, rapid tumor growth requires an increased influx of nutrients and efflux of waste products. The formation of new blood vessels to cope with this demand is frequently inadequate and hence results in characteristic tumor-associated phenomena such as hypoxia [23], high interstitial fluid tension [24] and low $\mathrm{pH}$ [25]. In addition, tumor-associated blood vessels are frequently leaky in nature [26] and exhibit morphological abnormalities such as a lack in pericyte coverage, a serpentine course, irregular branching, arterial-venous shunts and irregular perfusion (Fig. 1) [27,28]. Moreover, the lymphatic drainage of many solid tumors is often heavily impaired [29]. Combined with the production and remodeling of the ECM at the tumor margin [30,31], these anomalies lead to an increased interstitial fluid pressure within the tumor [32,33].

As first described by Matsumura and Maeda [34], the leaky properties of the tumor vasculature can be exploited to more selectively deliver biocompatible particles into solid tumors. This concept became known as the "enhanced permeability and retention (EPR) effect" [26]. To achieve successful EPR-driven drug delivery, the used particles mainly need long circulation times. Though distinct tumor accumulation is already seen as soon as 30 minutes, exploitation of the EPR effect requires drug delivery vehicles to circulate at least several hours in mice. This is obtained when the particles are prevented from clearance by the kidneys or the MPS, do not interact with blood vessels, are not immunogenic and remain intact [26]. The resulting drug retention in the tumor is reported to last several days to weeks [35]. In practice, the used particles should be smaller than $780 \mathrm{~nm}$ to pass through the pores of the tumor endothelium but larger than $40 \mathrm{kDa} / 5.5 \mathrm{~nm}$ to avoid their rapid renal clearance [36-38]. A frequently used strategy to prolong nanoparticle circulation time is to provide them with a shell of polyethylene glycol (PEG), a polymer known to reduce opsonisation and premature clearance by the MPS [39]. However, repeated administration of PEGylated agents can elicit an immune response leading to accelerated blood clearance due to formation of anti-PEG antibodies [40].

About three decades ago, the EPR effect sparked the emergence of

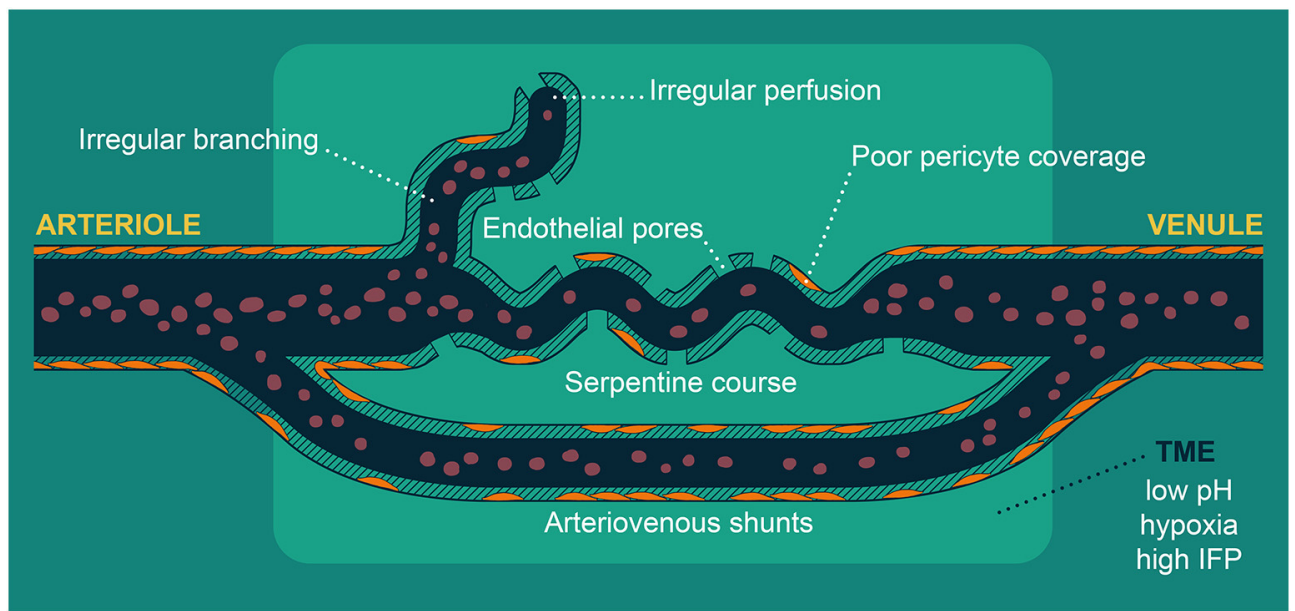

the nanoparticle research field which was heavily reliant on the "passive targeting" provided by this effect. Since then, many strategies have been devised to provide a complementary "active targeting" component to the used nanoparticles (i.e. adding specific ligands which target overexpressed receptors on cancer cells or in the TME) [36,41]. Despite the initial enthusiasm, this field is currently confronted with skepticism due to failure of providing relevant enhanced tumor accumulation in the clinic [42,43]. Moreover, the added benefit of targeting ligands are minimal compared to the physicochemical properties of nanoparticles $[36,44]$. A comprehensive literature review corroborated these findings and stated that even in mouse tumors, only $0.6 \%$ (median) of the injected nanoparticles reach the tumor via passive targeting [45]. Employing active targeting strategies modestly raised this value to almost $1 \%$, but no evolution in the delivery efficiency has been seen over the span of 10 years [45]. This setback is even more pronounced in humans and can be attributed to differences in tumor manifestation in preclinical animal models compared to tumor growth in humans [45]. The most notable differences relate to the relatively more heterogeneous nature of human tumors compared to experimental rodent tumors and include the smaller tumor-to-body size in humans, the absence of hyperpermeable vasculature, the high interstitial fluid pressure, the presence of avascular regions and the chaotic TME in human tumors $[42,46]$. These unfortunate developments highlight the limitations of animal models and emphasize the diverse nature of tumor biology. They also generate novel opportunities such as normalization of the tumor vasculature to increase tumor drug delivery [47-50]. In addition, novel studies such as the one by Sindhwani et al. (2020) suggest that almost all injected nanoparticles enter solid tumors via active endothelial transcytosis instead of EPR [51]. Although these mechanisms of nanoparticle transcytosis and their ability to specifically target tumors still need clarification, they bear the potential to elicit a paradigm shift in the nanoparticle research field [52].

Unlike small circulating compounds such as drugs, antibodies and nanoparticles, larger immune cells do not rely on passive diffusion to enter the TME but use their complex cellular machinery to navigate to and through the tumor-associated endothelium to occupy specific regions within the TME [53-57]. Consequently, immune cells potentially provide solutions to the obstacles that emerged in the field of targeted drug delivery. For example, immune cells do not require the presence of endothelial pores to initiate extravasation [58], are able to migrate into areas exerting high interstitial pressure and can even populate avascular regions deep inside solid tumors [59]. Furthermore, since tumor manifestation is often a form of unresolved chronic inflammation, immune cells are continuously recruited to this site $[60,61]$.
Fig. 1. Tumors display aberrant blood vessels. In normal blood vessels, arteries size down to arterioles and perfuse a capillary bed which then drains into venules of the venous system. A continuous layer of pericytes within the basement membrane encircles these small blood vessels. In contrast, the vasculature of solid tumors demonstrates a severely disarranged capillary bed with typical tortuous (serpentine) course, irregular branching and endothelial pores. Moreover, tumor blood vessels are poorly covered by pericytes and exhibit arteriovenous shunts that bypass certain regions of the capillary bed. 


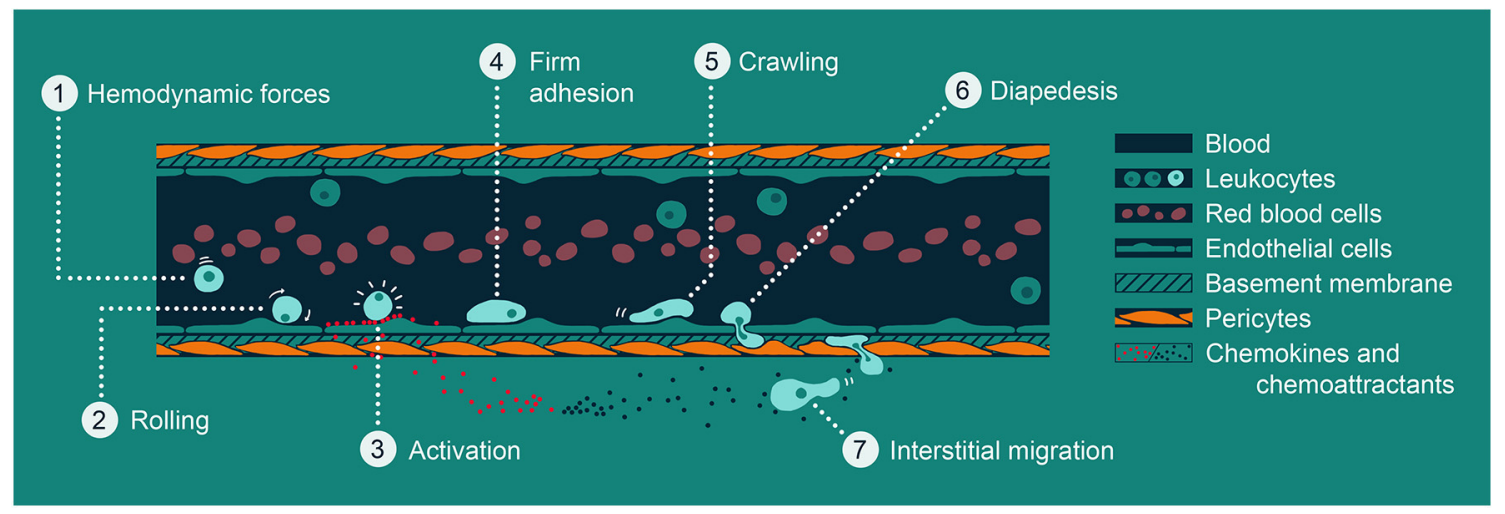

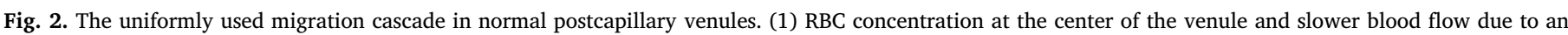

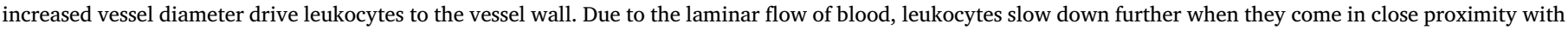

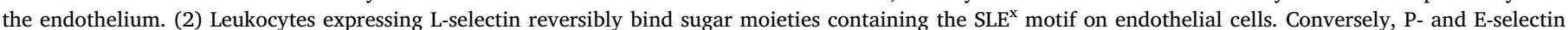

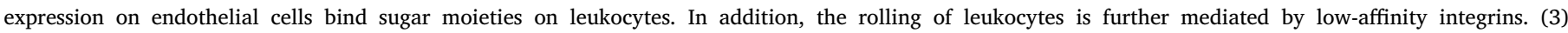

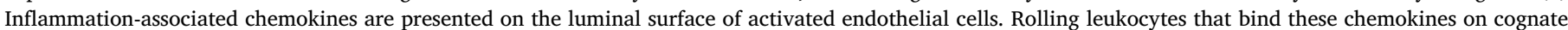

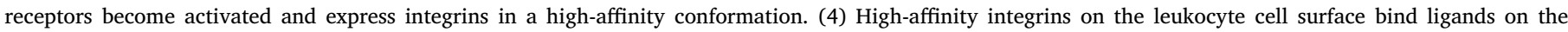

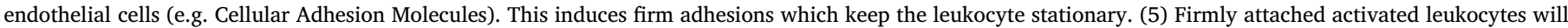

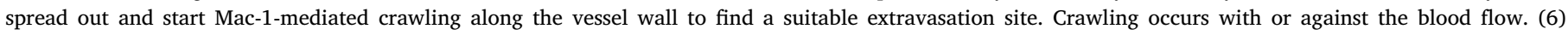

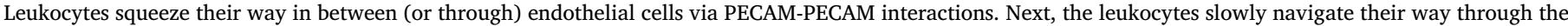

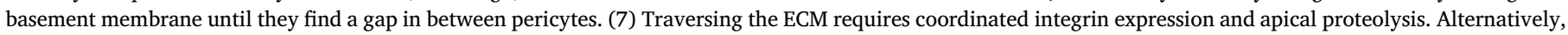

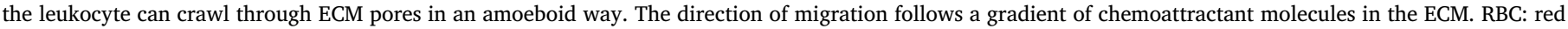
blood cells, SLE ${ }^{\mathrm{x}}$ : Sialylated Lewis X, Mac-1: Macrophage-1 receptor, PECAM: platelet endothelial cell adhesion molecule, ECM: extracellular matrix.

\section{How immune cells reach their target}

Circulating immune cells respond to inflammation-associated cues in a relatively uniform manner. However, some important distinctions exist between different immune cell populations that have an effect on their response time and trafficking patterns. For example, in contrast to granulocytes, naive lymphocytes are poorly responsive to inflammatory signals. They migrate efficiently to secondary lymphoid tissue where they first need to be activated before they acquire the capacity to be recruited to inflammation sites [62]. Immune cell trafficking occurs in sequential steps where each step is conditional for the next and thus, generates high targeting specificity (Fig. 2) [63]. These steps often take place in postcapillary venules, small veins with a diameter of about 50 $\mu \mathrm{m}$ which are located directly after the sites of increased vascular permeability during inflammation. The leakage of plasma and the wider lumen increase hemoconcentration, slowing the blood flow and allowing more interactions between the endothelium and leukocytes [64]. In addition, hemodynamic forces confine red blood cells (RBCs) to the center of the blood vessel, thereby "marginating" other large particles such as immune cells to the vessel walls [65].

Passing immune cells that come into contact with the endothelial layer loosely bind sugar moieties, thereby inducing a reversible leukocyte rolling along the endothelial surface lasting several seconds to minutes $[66,67]$. This first step in the migration cascade is mediated by selectins (CD62), cell surface molecules that bind distinct terminal carbohydrate residues (a tetrasaccharide motif known as "sialylated Lewis X") of glycoproteins or glycolipids in a calcium-dependent fashion $[62,66]$. Selectins are composed of three families named after the cell type on which they were originally identified: E-selectin on activated endothelium, P-selectin on activated platelets, but also on activated endothelium and L-selectin constitutively on lymphocytes, but also expressed on hematopoietic stem/progenitor cells $[62,66]$. The carbohydrate selectin ligands are present on endothelial cells and other leukocytes, thus facilitating leukocyte-endothelium or leukocyte-leukocyte interactions [68]. Of all the available ligands, P-selectin glycoprotein ligand 1 (PSGL-1) appears to be the main ligand for all these selectins [69]. The non-covalent bonds forming between the rolling leukocyte and the vessel wall must counteract the shear stress, the horizontal force and torque exerted by the blood flow [70].
Interestingly, this involves selectin-associated "catch bonds" which require a certain amount of shear stress for leukocytes to attach to the endothelium and may prevent inappropriate leukocyte aggregation or extravasation during vascular stasis [68]. After reaching a maximal shear stress value, selectin-ligand interactions change from "catch bonds" to regular "slip bonds" [70]. This delicate balance makes the cell pivot over the remaining binding sites and hence roll to a position further downstream at about $10 \mu \mathrm{m} / \mathrm{s}$. In addition to mediating reversible cell-cell adhesion, selectin binding also activates intracellular signaling pathways leading to e.g. $\beta 2$-integrin activation associated with intermediate affinity [71,72]. Consequently, selectin-mediated rolling further reduces rolling velocities to about $5 \mu \mathrm{m} / \mathrm{s}$ by facilitating conformational changes in integrins and thus providing more opportunities for rolling cells to encounter endothelium-bound chemokines [72,73]. In turn, the encountered inflammation-associated chemokines which are displayed on the endothelium surface will further stimulate integrin activation that mediates leukocyte arrest and subsequent transendothelial migration [64].

Integrins are transmembrane cytoskeleton-linked heterodimers consisting of paired $\alpha$ and $\beta$ subunits. These subunits assemble into many different receptors with different binding properties and tissue distribution [74]. Essentially all nucleated cells express integrins [73], but the 32 - or CD18-integrins are only expressed on leukocytes [73,75]. For example, lymphocyte function-associated antigen 1 (LFA-1, $\alpha \mathrm{L} \beta 2$ or $\mathrm{CD11a} / \mathrm{CD} 18$ ) is expressed on all leukocytes and is the predominant integrin on lymphocytes while macrophage- 1 (Mac-1, CR3, $\alpha \mathrm{M} \beta 2$ or $\mathrm{CD} 11 \mathrm{~b} / \mathrm{CD} 18)$ dominates on myeloid leukocytes [75]. Integrin activation induces rapid (i.e. within $1 \mathrm{~s}$ ) conformational changes from a completely bent form with low ligand affinity to an intermediate or fully extended form with very high ligand affinity [74,76,77]. Most integrins typically bind a wide variety of ligands, making these receptors essential in many cell-cell and cell-matrix interactions [74]. Though integrins can be involved in the rolling of leukocytes [78], they are mostly important for inducing firm adhesions. Conformational change of integrins is initiated by other cell surface receptors (e.g. selectins, chemokine receptors, Toll-like receptors or the $\mathrm{T}$ cell receptor) and occurs through cytoplasmic activation of the integrin $\beta$ subunits $[75,79]$. Once activated, the integrins can bind their endothelial ligands such as members of the immunoglobulin superfamily (e.g. ICAM-1, 
VCAM-1 and MAdCAM-1) [75]. At inflammatory sites, this is followed by cell spreading and Mac-1-mediated crawling along the vessel wall to find suitable extravasation sites [80,81].

Most of the immune cells that pass through the vasculature of the inflammation site do not roll, most of those that roll do not adhere and most of the adherent immune cells do not extravasate [82]. However, the commitment of immune cells to initiate extravasation marks a certain "point of no return" [82]. Transendothelial migration or diapedesis is initiated by clustering of leukocyte integrins with their endothelial ligands and occurs in between (para-) or to a lesser extent through (trans-) endothelial cells $[83,84]$. The resulting actin-rich endothelial docking structures then form a tight ring around the transmigrating leukocyte [64]. VE-cadherin (CD144) normally connects endothelial cells at adherens junctions, but is cleared from the site of transmigration to form a gap. In addition, a dynamic vesicle complex known as the lateral border recycling compartment at the intercellular junctions between endothelial cells has to assemble around the extravasating leukocyte and continuously recycle to facilitate transendothelial migration [64]. This requires PECAM-PECAM or CD31 interactions between the leukocytes and the endothelium as well as an essential increase of intracellular calcium in the endothelial cells [64]. Once the immune cell has successfully crossed the endothelial layer within less than 5 minutes, it must also find a way through the basal lamina. In general, this can take up to 15 minutes as the leukocyte has to navigate its way to areas with less collagen IV and laminin 511 deposition [85-88]. These areas frequently occur at gaps in pericyte coverage and squeezing through these gaps involves many of the molecules seen for transendothelial migration $[64,89]$. In addition, leukocytes require deformable nuclei to traverse these narrow pores and can rely on protease-mediated remodeling of the basement membrane to clear the path [89].

After immune cells have penetrated the dense sheet-like basement membrane, migration through the rest of the interstitial mesh-like ECM starts. The ECM is a highly complex organ-specific network of fibrillary and non-fibrillary collagens, glycoproteins and proteoglycans assembled in supramolecular structures which provide structural support, but also bind and regulate the distribution of soluble factors such as growth factors and chemokines [87,90]. Immune cell migration through the ECM is achieved by coordinated expression of certain collagen-binding and -sensing integrins [87]. In addition, secreted and membrane-bound proteases e.g. matrix metalloproteinases can help in clearing the path [91]. In turn, this localized proteolysis of the extracellular matrix exposes more integrin ligands, cytokines and chemokines which elicit a combined directional response in the migrating leukocyte [92,93]. Active breakdown of the ECM and integrin-mediated attachment is not always a requirement as leukocytes can also navigate through pre-existing matrix gaps in an amoeboid way independent of matrix-degrading enzymes or even integrins [94]. Nonetheless, cell type-specific limitations in nuclear stiffness impose protease activity as ECM pores are typically smaller than $1 \mu \mathrm{m}[87,95]$.

Chemotaxis is the movement of an immune cell in response to chemical substances present in tissues (chemoattractants). Secreted cytokines that elicit such chemotaxis are termed chemokines and typically bind cellular G protein-coupled receptors [96]. They are subdivided into four classes based on the spacing between the first 2 cysteine (C) residues at the amino terminus. These 2 cysteines are separated by either zero, one or three amino acids in respectively $\mathrm{C}$, $\mathrm{CXC}$ and $\mathrm{CX}_{3} \mathrm{C}$ chemokines. $\mathrm{C}$ chemokines have only one cysteine at the $\mathrm{N}$-terminus [97]. Chemokines are constitutively expressed under homeostatic conditions to maintain immune surveillance, but activated cells can also produce inflammatory chemokines to recruit additional immune cells to inflamed tissues [96].

Leukocytes first encounter chemokines, produced by interstitial or vascular cells, while rolling on the endothelial surface at sites of inflammation [98]. This interaction activates integrins, thereby initiating transendothelial migration. Upon arriving in the ECM, immune cells sense gradients of chemotactic cues in a cell type-specific manner and migrate towards the region of highest concentration [96,98]. As many chemoattractants influence the chemotaxis simultaneously, migrating immune cells have the capacity to hierarchically prioritize certain chemokine gradients [96]. Interestingly, migrating immune cells may also release chemokines themselves, allowing other immune cells to follow their trail [99]. To establish a gradient, soluble chemokines need to diffuse away from their source. To prevent chemokines from washing away, they are immobilized in the ECM and on the luminal surface of endothelial cells via glucosaminoclycans $[96,100]$. Nevertheless, dislodged chemokines are also detected in the blood during inflammation. These blood-borne chemokines are suspected of activating immune cells off-site and by doing so, causing immune cell accumulation in organs such as the lungs and the liver [96].

\section{Hijacking cells for drug delivery purposes}

Immune cells are continuously recruited to establish a TME that can be either anti- or pro-tumorigenic. Tumors can be pathologically categorized according to the relative presence of these different recruited immune cell populations and the character of their inflammatory response [101-103]. For example, tumors that are heavily populated by T lymphocytes are considered immunologically "hot" whereas "cold" tumors are characterized by an absence of these cells. In between these 2 extremes, we find "infiltrated-excluded" TMEs which are heavily populated by lymphocytes at their periphery but lack cytotoxic lymphocytes (CTLs) in the tumor core [104]. In addition, "infiltrated-immunosuppressed" TMEs contain a low number of lymphocytes with suppressed effector function in the tumor core [101]. Tumor eradication requires combined innate and adaptive immune responses after successful recognition of the malignant cells. Likewise, tumor growth also relies on hematological cells to establish an immune-suppressive TME. Therefore, both scenarios require immune cells to be recruited [101]. This premise forms the main argument for using cells as drug delivery vehicles. In addition, due to the ubiquitous nature of this approach, it has the potential to result in targeting strategies that are widely applicable in cancer patients.

Like other drug carriers, cells can be used to protect their therapeutic cargo from immune recognition and degradation, thereby prolonging its circulation time [105]. In addition, the inherent migration capacity of immune cells can be used to specifically target inflammatory regions and the TME (Fig. 3). Even metastases can potentially be targeted using this approach, since they are also associated with increased immune cell infiltration [106]. Unlike the diffusiondriven delivery used by other carriers (e.g. liposomes, synthetic polymers and protein-DNA complexes), cells can truly actively penetrate solid tumor tissues, even when they are characterized by high interstitial pressure. Moreover, when DNA or RNA are used as launching platforms for therapeutic proteins, such cellular carriers can effectively be used as local production factories [105]. The possibilities of using bioengineering tools to develop and iteratively improve molecular sensors and switches further accentuates the advantages and future potential of intelligent cell delivery vehicles [107-110].

\section{Red blood cells}

In theory, red blood cells (RBCs or erythrocytes) are ideal drug carriers with the drugs either residing inside the RBCs or adsorbed to their surface. These simple cells are readily available in vast quantities, are very biocompatible even in allogeneic settings and are easily stored [111]. RBCs have virtually unrestricted access to vascularized tissues because of their relatively small size $(7 \mu \mathrm{m}$ diameter and $2 \mu \mathrm{m}$ thick) and capacity to deform (Table 1 ). In humans, they travel through the circulatory system every 20 seconds and exhibit a lifespan of about 3-4 months in circulation while mouse RBCs have a lifespan of only 1 month [105,112-114]. Due to the lack of a nucleus, RBCs can be 


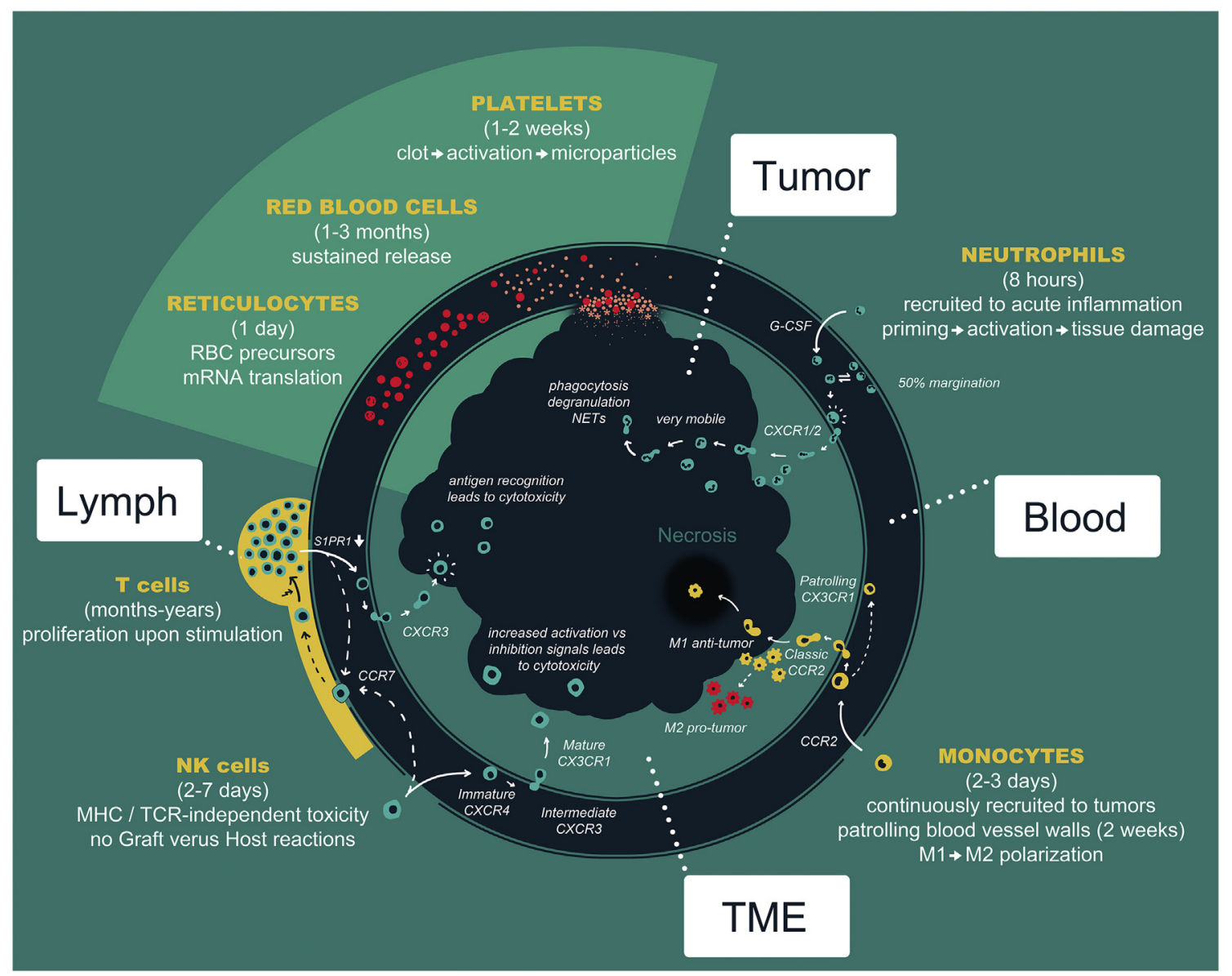

Fig. 3. Blood circulating cells as drug delivery vehicles. Non-immune cells such as reticulocytes, red blood cells and platelets can be used for intravascular release of drugs but do not actively migrate to the interstitial space (green sector). Immune cells such as neutrophils, monocytes, NK cells and T cells traverse the blood (and lymphatic) vessels before initiating transmigration into the TME and tumor tissue. The circulation time, the most distinctive properties and main chemoreceptors for each cell type is depicted. RBC: red blood cells, MHC: major histocompatibility complex, TCR: T cell receptor, S1PR1: Sphingosine 1-phosphate receptor 1.

viewed as biological microparticles that can be used to compartmentalize drugs, e.g. to avoid renal clearance [14]. However, their size and lack of migration machinery confines the use of RBCs to intravascular applications such as circulating bioreactors for enzymes, as reservoirs for slow drug release, as carriers for imaging agents and as "supercarriers" for nanoparticles [14,113]. Alternatively, RBC precursors (reticulocytes) contain translation machinery and can therefore be exploited as production factories for mRNA-based therapeutics [115]. Some degree of targeting of the MPS is described e.g. via cell surface modifications which mimic damaged or senescent RBCs [116,117].
Since a large portion of the MPS resides in the spleen and the liver, the MPS-mediated clearance of RBC drug carriers is sometimes seen as active targeting of these organs [111]. Nevertheless, since virtually all circulating particles, debris and compromised cells are eventually cleared by cells of the MPS, the added benefit of using RBCs for this goal over liposomes can be questioned [118].

Most drug delivery strategies try to minimize RBC elimination [113]. Apart from avoiding membrane alterations due to the drug loading protocols, CD47 as a macrophage "do not eat me" signal or complement inhibitory molecules can be introduced in the RBC

Table 1

Main properties of blood cells that have been considered as drug delivery vehicles.

\begin{tabular}{|c|c|c|c|c|}
\hline Cell type & Size $(\mu \mathrm{m})$ & Lifespan & Main chemokine/receptor & Remarks \\
\hline $\mathrm{RBC}$ & $6-7$ & 3-4 months (Mouse: 1 month) & - & only for intravascular use, no nucleus, no activation \\
\hline Platelet & $1-3$ & 9 days & - & Interesting for injuries involving hemostasis, no nucleus \\
\hline Neutrophil & $7-11$ & $6-8$ hours $\left(\mathrm{t} \frac{1}{2}\right)$ & CXCL8/CXCR1-2 & Widely applicable, rapid recruitment, collateral damage \\
\hline Eosinophil & $12-17$ & $8-12$ hours & CCL11/CCR3 $^{\mathrm{a}}$ & Collateral damage, allergens \\
\hline Basophil & $5-7$ & $1-2$ days & CCL11/CCR $^{\mathrm{a}}$ & Collateral damage, allergens \\
\hline Monocytes & $10-18$ & $\begin{array}{l}\text { Ly6C }{ }^{\text {high }}: 17 \text { hours }\left(\mathrm{t} \frac{1}{2}\right) \\
\text { Ly6C }^{\text {low }}: 2-14 \text { days }\left(\mathrm{t} \frac{1}{2}\right)\end{array}$ & $\begin{array}{l}\text { Ly6C } \text { high: }^{\text {CCL2/CCR2 }} \\
\text { Ly6C }^{\text {low: }} \text { CX3CL1/CX3CR1 }\end{array}$ & $\begin{array}{l}\text { Widely applicable, versatile functions, penetration into necrotic tumor core } \\
\text { React to ex vivo manipulation }\end{array}$ \\
\hline $\mathrm{T}$ cell & $8-10$ & 2 weeks after activation & PSGL-1/CXCR3 & Local proliferation and memory cells, cytotoxic upon antigen recognition \\
\hline NK cell & 10 & 1 week & CXCL8/CXCR1 $1^{\mathrm{b}}$ & MHC-independent cytotoxicity \\
\hline
\end{tabular}

${ }^{\text {a }}$ Eosinophils and basophils use very similar chemokine receptors but both cell types rely on specific combinations of receptors to achieve successful recruitment into tissues and their main receptor CCR3 binds a large array of ligands [151].

b CXCR1 is an example of a chemokine receptor that can only be found on the cytotoxic NK cell subset. 
membrane. Moreover, RBC "immune-camouflaging" via PEGylation offers some interesting prospects [113,119]. Accumulation of RBCs in specific organs can be achieved by incorporating magnetic materials into drug-loaded RBCs and subsequently applying an external magnetic field on the organ of interest [120]. Similar strategies include the induction of microvessel rupture through targeting microbubbles via ultrasound [121] or coating of the RBC surface with targeting moieties such as antibodies [122]. However, since hydrodynamic forces drive RBCs to the center of the vascular lumen and RBC-endothelial interactions are minimized by the $0.5 \mu \mathrm{m}$ thick endothelial glycocalyx [123], the use of targeting moieties may not be efficient. It is currently unclear how these approaches can be applied to situations where the pathological region is unknown such as in metastatic disease.

Within the context of cancer therapy, RBC drug carriers can provide the same benefits as liposomal drug formulations such as Doxil ${ }^{\circledast}$, a commercial PEGylated liposomal formulation of doxorubicin, but with a decreased risk of mounting immune responses [124,125]. Interestingly, although doxorubicin-loaded RBCs demonstrated virtually equal tumor targeting compared to free doxorubicin, other differences in biodistribution were noticed. Indeed, RBC-doxorubicin demonstrated increased accumulation in the liver, the spleen and the lungs while free doxorubicin resulted in a higher accumulation in the heart and skin of tumor-bearing mice. Despite the prolonged release of doxorubicin from the circulating RBCs that resulted in a significantly decreased tumor volume, less cardiotoxicity and myelosuppression was seen [124]. Therefore, the effects of RBC-mediated drug delivery seem to be twofold: sustained release which maintains drug concentrations within the therapeutic window and skewing biodistribution towards organs of the MPS. However, a main limitation is that RBCs cannot be used to actively deliver drugs to extravascular targets.

\section{Platelets}

Platelets or thrombocytes are smaller $(1-3 \mu \mathrm{m})$ than RBCs and only circulate for about 9 days followed by their clearance via the MPS [126]. They have a key role in blood clotting and secrete bioactive molecules such as growth factors, chemokines, cytokines and matrix metalloproteinases upon their activation. Moreover, they are also involved in inflammation, wound healing and tumor progression [126-128]. Activation takes place when circulating platelets encounter damaged endothelial walls, where they change shape, shed off platelet microparticles and aggregate to form clots through the interaction with fibrin [129]. Pathological release of excessive platelet activators can cause platelets aggregation within intact blood vessels or thrombosis [126]. Like RBCs, platelets do not possess a nucleus and cannot actively penetrate tissues. Nevertheless, their advantage as drug delivery vehicles is that activation allows them to acquire more selectivity compared to RBCs. As such, they can be used to selectively deliver therapeutic cargo into platelet-activating tumors [130,131]. For example, platelets have been loaded with doxorubicin to treat lymphoma-bearing mice [132]. Similar to RBCs, this targeting strategy maintained the tumor inhibiting effect of doxorubicin while avoiding its cardiotoxicity $[132,133]$. Perhaps the most efficient strategy to utilize platelets for drug delivery is post-surgical treatment because this medical intervention is always associated with bleeding and hence, hemostasis. In this context, a recent study demonstrated substantially increased antiPDL-1 efficacy through platelet-mediated drug delivery against residual melanoma cells after surgical removal of the primary tumor [134,135]. Anti-PDL-1 antibodies were conjugated to the cell surface allowing them to be dispersed on platelet-derived microparticles after activation of the carrier platelets. This approach was also successfully demonstrated after thermal ablation of the primary tumor [136].

\section{Myeloid cells}

Immune cells of the myeloid lineage mostly comprise cells involved in the innate immune system (e.g. granulocytes, monocytes, macrophages, dendritic cells), but provide an essential bridge to the adaptive immune system as well. They are implicated in all inflammation processes and form important members of the TME. The modern view on cell function incorporates a spectrum of functions ranging from immune stimulatory to -suppressive, depending on the input the cell receives [137]. In this regard, solid tumors frequently produce soluble factors which favor the production and recruitment of (immature) myeloid cells to the TME [138]. Paradoxally, these innate immune cells are submerged in an array of tumor-derived factors, instructing them to adopt phenotypes that are favorable for tumor survival, progression and eventually metastasis [138]. Therefore, myeloid cells are essential components in establishing an immunosuppressive TME reminiscent of a state of chronic inflammation [139,140]. Due to the plastic nature of myeloid cells in such an environment i.e. the ability to switch phenotypes, much debate arises concerning their classification [141,142]. Most of these cells derive from circulating immune cells which were recruited via the classic migration cascade. Of note, myeloid cells are also suspected of shaping the "pre-metastatic niche", indicating that their recruitment can even precede the arrival of metastatic tumor cells $[143,144]$. Besides the large amount of chemotaxins that recruit multiple immune cell populations, the occurrence of post-translationally modified (e.g. glycosylation) chemokines in the TME was reported $[145,146]$. These altered chemokines can demonstrate a change in receptor specificity and affinity, thereby selectively recruiting certain leukocyte populations while dismissing others [147]. It is therefore possible that additional selectivity can be obtained by focusing on those populations that specifically react to these tumor-associated altered chemokines. Given the high number of chemotaxins and corresponding receptors involved in leukocyte recruitment, this will most likely require elaborate integrated analysis to predict their combined effect. Still, myeloid cells have the inherent capacity of trafficking to solid tumors. Those myeloid cell populations that have been reported as drug delivery vehicles will be discussed below.

\subsection{Granulocytes}

Bone marrow-derived granulocytes are a group of innate immune cells characterized by an irregular nucleus and the presence of granules in their cytoplasm. They are typically further subdivided into 3 families: neutrophils, eosinophils and basophils. Neutrophils have historically been extensively studied due to their relative abundance i.e. $40-75 \%$ of the total leukocyte count in human blood and their central role as "first responders" in most inflammatory situations [148]. The vast amount of available data on their biodistribution and migration properties provides us a template to which other immune cell populations can be compared.

\subsection{Neutrophils}

In the bone marrow, granulocyte colony stimulating factor (G-CSF) is the main stimulator of neutrophil differentiation, proliferation and release [149]. Neutrophils $(7-11 \mu \mathrm{m})$ are kept in the bone marrow through the interaction with chemokine SDF-1, which is constitutively expressed by bone marrow stromal cells. Binding of SDF-1 on neutrophil receptor CXCR4 then modulates integrin-ligand binding VLA-4/ VCAM-1 [150]. G-CSF inhibits this interaction by down-regulating the expression of both SDF-1 and CXCR4, resulting in the release of neutrophils [149]. After leaving the bone marrow, neutrophils circulate in the blood with a short half-life of only 6-8 hours. In the blood they either slowly transit through intravascular "margination pools" such as the liver, the spleen, the bone marrow and the lungs, get cleared from the circulation or enter inflamed tissues $[149,151]$. About $50 \%$ of the intravascular neutrophils will reversibly bind/roll along the endothelium of postcapillary blood vessels [152-155]. This marginated pool is in dynamic equilibrium with the circulating pool, a balance that 
can be manipulated to mobilize neutrophils back into circulation. Of note, organ sequestration of cells also includes irreversibly bound cells, trapped cells (e.g. by the MPS) and cells that migrated in the interstitium [155]. Therefore, calculating the size of the marginated pool in an organ is challenging, but most reports indicate that besides the liver, especially the spleen and the bone marrow contain many of these marginated neutrophils [155,156]. Moreover, a large portion of the marginated neutrophils reside in the lungs, most likely due to the high number of capillaries which all cells ejected from the heart have to squeeze through [155,157-159]. Hence, increasing cardiac output via e.g. adrenaline forces neutrophils into circulation $[157,158]$.

Sequestration in a particular organ also depends strongly on the maturation and activation status of the neutrophils [160]. The majority of immature mouse neutrophils isolated from the bone marrow home back to the bone marrow after infusion, but can later be redistributed to sites of inflammation, while mature neutrophils localized equally between the liver and the bone marrow after injection, with no observed remobilization to an inflammation site [160]. In fact, bone marrow can act as a clearance organ for senescent neutrophils where they are phagocytized by macrophages, which in turn produce G-CSF to stimulate the production and release of other neutrophils [156]. In contrast, apoptotic neutrophils are mainly cleared by the liver Kupffer cells after exposing phosphatidylserine on their cell surface [161]. Noteworthy, inflammation-induced remobilization also causes neutrophils to accumulate in the lungs, which sometimes leads to acute lung injury during inflammatory states [162,163].

Activation of neutrophils occurs in 2 stages. Upon encountering certain inflammation-associated factors (e.g. cytokines, chemokines, bacterial and viral products), neutrophils are first 'primed' after which they can demonstrate an enhanced activation by interaction with e.g. pathogen- or damage-associated molecular patterns (PAMPs or DAMPs) $[151,164]$. Priming induces cytoskeleton changes, making the neutrophils more rigid and consequently more prone to accumulating in capillary beds $[159,165,166]$. It also induces many other phenotypic changes, including adhesion to endothelium through increased expression and activation of $\beta 2$ integrins, extravasation, increased responsiveness to chemokines (CXCL8 (IL-8) in particular) [167] and resistance to apoptosis $[164,168,169]$. Thus, neutrophils that underwent transendothelial migration are by definition primed [170]. Interestingly, circulating neutrophils can also be de-primed in the pulmonary blood vessels by currently unknown mechanisms, and this reversible activation state influences the kinetics and biodistribution of neutrophils $[166,169]$. Thus, relatively nonresponsive neutrophils become primed after encountering a first stimulus after which they become fully activated upon encountering a second stimulus. This state is characterized by phagocytic activity, degranulation and production of neutrophil extracellular traps (NETs) [171].

Despite their abundance (50-70\% of white blood cells in humans, $10-30 \%$ in mice), rapid recruitment and capacity to fight cancer cells $[172,173]$, neutrophils are rarely used as cell delivery vehicles because of their reactive nature and short lifespan. Moreover, neutrophils are notoriously known to cause substantial collateral damage to healthy tissues after degranulation [174]. Nevertheless, to combat antibiotic resistance, Wendel et al. (2015) devised an interesting strategy involving the encapsulation of chlorhexidine by dead bacteria used as stable liposome-like drug carriers [175]. These bacteria were loaded into neutrophils, infused in mice with liver abscesses and their subsequent infiltration into the liver significantly reduced the bacterial burden. Unfortunately, this study did not report biodistribution data. Moreover, the absence of a chlorhexidine only-control prevents interpretation of the added effect of using neutrophils as delivery vehicles. Such a control was probably omitted from the study due to the toxic properties of this topical antiseptic agent.

Overall, the short lifespan of neutrophils might enable to use these innate immune cells as delivery vehicles in acute therapeutic settings, provided that their off-target effects are genuinely restrained. In addition, the concept of loading therapeutic agents (e.g. via nanoparticles) in immune cells within a patient is definitely worth pursuing as it eliminates the need for collecting, isolating, loading and reinfusing immune cells [176]. In this regard, Chu et al. (2017) demonstrated that intravenously injected anti-CD11b-coated nanoparticles are taken up by neutrophils which then accumulate in tumor tissue after induction of acute inflammation [177]. In contrast, only low levels of accumulation was seen upon injection of PEGylated nanoparticles or when no acute inflammation was induced [177]. Similarly, neutrophils were used to deliver paclitaxel-loaded liposomes to surgically resected gliomas [178].

\subsection{Eosinophils and basophils}

After release from the bone marrow by IL-5, eosinophils $(12-17 \mu \mathrm{m})$ circulate only $8-12$ hours in blood, where they comprise $1-3 \%$ of the total leukocyte count and rely heavily upon CCR3 for their recruitment into tissues(Table 1) [179,180]. Eosinophils have many homeostatic functions, but can also be recruited in response to inflammation where they are best known for their IL-5-mediated interactions with Th2 lymphocytes during parasitic infections and in response to allergens [179]. Inflammation-induced degranulation of these cells is typically associated with cell toxicities and disruption/remodeling of the ECM [179]. They are also implicated in establishing the TME of solid tumors, demonstrating their potential use as drug delivery vehicles [181]. Eosinophil recruitment to tumors occurs in all stages of tumor growth and independently from Th2 lymphocytes. Their accumulation is mainly restricted to the fibrous tumor capsule and to areas of necrosis [182]. The capacity of adoptively transferred bone marrow-derived eosinophils to be efficiently recruited to inflammatory lungs upon allergeninduction hints at a potential therapeutic use in this specific organ [183]. However, it has been demonstrated that this type of inflammation is not restricted to eosinophils, but also recruits neutrophils and lymphocytes [184].

Basophils (5-7 $\mu \mathrm{m})$ represent the lowest fraction of leukocytes in the blood $(<1 \%)$. They have an estimated lifespan of $1-2$ days and are mostly implicated in inflammation reactions associated with allergies and parasites (Table 1) [180,185,186]. However, this stereotypical view is currently discarded in favor of a complex immunomodulatory and tissue remodeling function that is strongly dependent on its environment $[185,187]$. Their capacity to produce IL-4 and subsequently elicit Th2 cell responses after adoptive transfer suggests therapeutic potential of these cells to modulate immune responses [188]. Like eosinophils, recruitment of basophils into tissues uses multiple chemokine receptors and largely relies on the same chemokine receptors [189].

Eosinophils and basophils have not been explored as drug delivery vehicles most likely due to their low presence in the blood, and hence the problems associated with obtaining enough cells. Moreover, these cells can potentially cause extensive tissue damage [182,186]. Nevertheless, since eosinophils and basophils are specialized cells implicated in specific disease situations, they might have therapeutic potential in future delivery strategies. Alternatively, parts of their migration machinery such as selected chemokine receptors could also be incorporated in other cells to acquire their specific tissue tropism.

\subsection{Monocytes and macrophages}

A population of self-maintaining tissue-resident macrophages and classical dendritic cells (DCs) are established before birth. In addition, bone marrow-derived cells of the monocytic lineage are continuously produced to replenish and assist these cells in maintaining tissue integrity after birth $[190,191]$. The macrophage-DC precursor (MDP) or monoblast, a common founder cell residing in the bone marrow, differentiates into dedicated DC precursors and "common monocyte progenitors" [192,193]. Monocyte development mostly relies on the interaction of M-CSF or CSF1 with its receptor M-CSFR or CD115 and 
monocytes subsequently require CCR2 to exit the bone marrow [194,195]. The postnatal bone marrow constitutes the main production site of monocytes, but relocation of hematopoietic stem cells and progenitor cells to the spleen can form supplementary sites of extramedullary hematopoiesis [196].

Monocytes represent about $10 \%$ of the circulating leukocytes in human and $4 \%$ in mice. They comprise a highly dynamic cell population that is vital in ensuring rapid injury detection, inflammation and resolution. Like neutrophils, a substantial number of intravascular monocytes can be found in marginal pools within organs such as the lungs and the spleen $[197,198]$. Again, this marginated monocyte pool is in dynamic equilibrium with circulating monocytes and can be mobilized on demand [197,199]. Multiple monocyte populations are found within the blood vessels. In mice, these populations can be discriminated via their level of Ly6C expression, a cell surface marker variably expressed by many immune cell populations except B cells [200]. The Ly6C $\mathrm{C}^{\text {high }}\left(\mathrm{Gr}-1^{+}\right)$monocytes also highly express the chemokine receptor CCR2 and are the first subset of monocytes to be released from the bone marrow [200]. Conversely, Ly6 $\mathrm{C}^{\mathrm{low}}$ monocytes typically express the chemokine receptor CX3CR1 [201]. Cell surface phenotyping consequently reveals two distinct mouse monocyte populations: "immature", "classical" or "inflammatory" Ly6C Chigh/ $\mathrm{CX} \mathrm{CR} 1^{\mathrm{mid}} / \mathrm{CCR} 2^{+} / \mathrm{CD} 2 \mathrm{~L}^{+} / \mathrm{CD} 43^{\text {low }}$ monocytes, and "mature", "pa-

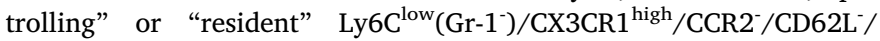
CD43 high monocytes (Table 2) [194]. The Ly6 Chigh $^{\text {he }}$ are termed "classical" because they typically enter inflamed tissues to further differentiate into peripheral mononuclear phagocytes i.e. macrophages or monocyte-derived DCs [194]. Of note, the murine Ly6C $\mathrm{C}^{\text {high }}$ monocyte subset is considered to be the precursor of smaller Ly6 $\mathrm{C}^{\text {low }}$ monocytes through an intermediary Ly6C $^{\text {int }}$ population [193,202]. Ly6C high monocytes can even home back to the bone marrow before maturing into Ly6C ${ }^{\text {low }}$ monocytes [203]. The latter can be seen as terminally differentiated blood-resident macrophages that survey endothelial integrity, hence they are termed "patrolling" monocytes [190,194,202]. While the circulatory half-life of Ly6 $\mathrm{C}^{\text {high }}$ monocytes is estimated at about 17 hours (Table 1), intravascular Ly6 $\mathrm{C}^{\text {low }}$ monocytes exhibit far longer half-lives ranging from 2 days up to 2 weeks [190].

Like their mobilization from the bone marrow, recruitment of Ly6C $\mathrm{C}^{\text {high }}$ monocytes to sites of inflammation (including in the central nervous system) is highly dependent upon the CCL2-CCR2 axis [204]. There, these inflammation-induced monocytes transiently assist the self-maintaining pool of tissue macrophages [194]. Dependent on the context of the microenvironment, monocyte-derived macrophages subsequently polarize to a spectrum of cells between 2 opposing phenotypes: the classically-activated or pro-inflammatory "M1" macrophages versus the alternatively-activated or anti-inflammatory, tissue repairing, "M2" macrophages [205]. It is currently unclear whether some of these monocyte-derived macrophages can further establish in the tissues through self-maintenance [194]. Nevertheless, it has been demonstrated that tissue macrophages can proliferate at low levels during steady-state and that their proliferative capacity is enhanced through environmental cues such as IL-4 and M-CSF [206-208]. This

Table 2

Commonly used cell surface markers of mouse and human blood cells.

\begin{tabular}{lll}
\hline Cell type & Mouse & Human \\
\hline RBC & CD235a, TER-119 & CD235a \\
Platelet & CD41, CD62P & CD42b, CD62P \\
Neutrophil & CD11b, Ly6G & CD15, CD16, CD66b, MPO \\
Eosinophil & CD11b, Siglec-F & CD11b, Siglec-8 \\
Basophil & CD200R3 & CD123 \\
Monocytes & CD11b, CD115, Ly6C, CCR2, CD62L & CD14, CD16, CD64 \\
T cell & CD3, CD25 ${ }^{\mathrm{a}}, \mathrm{CD} 69^{\mathrm{a}}$ & $\mathrm{CD} 3, \mathrm{CD} 25^{\mathrm{a}}, \mathrm{CD} 69^{\mathrm{a}}$ \\
NK cell & CD3(-), CD11b, CD27 & CD3(-), CD56, CD16 \\
\hline
\end{tabular}

a These markers are only present after cell activation. has also been observed for monocyte-derived macrophages in the gut and in atherosclerotic plaques [209,210].

As mentioned earlier, to successfully migrate from the bone marrow into inflamed tissues, Ly6 $\mathrm{C}^{\text {high }}$ monocytes rely on L-selectin-mediated rolling and CCR2-mediated activation and chemotaxis based on CCL2 and CCL7 gradients [193,211-213]. In addition to these well-known migration receptors, monocyte recruitment further involves many other integrins and chemokine receptors such as CCR1 and CCR5, a comprehensive list is provided in the excellent review by Shi and Pamer (2011) [214]. In contrast to these "inflammatory" Ly6 $\mathrm{C}^{\text {high }}$ monocytes, Ly6C ${ }^{\text {low }}$ monocytes do not express L-selectin nor CCR2 but primarily rely on CX3CR1 and $\beta 2$-integrins such as LFA-1 which engage with endothelial ICAMs [193,213]. Instead of rapidly rolling, steady state Ly6C ${ }^{\text {low }}$ monocytes continuously "crawl" over relatively long distances on the luminal side of mouse blood vessels i.e. at about $12 \mu \mathrm{m} / \mathrm{min}$, independent of blood flow direction [193]. Interestingly, the ideal location of Ly6 $\mathrm{C}^{\text {low }}$ monocytes makes them swiftly $(<1$ hour) extravasate into tissues upon induction of inflammatory stimuli, a response found to precede that of neutrophils [193].

Intravenous injection of mouse macrophages results in a typical distribution pattern [215]. This pattern is characterized by rapid disappearance from the circulating pool with an initial arrest in the lungs, followed by redistribution to mainly the spleen and the liver [215,216]. When a distant site of inflammation is present e.g. in the footpad, specific accumulation of a small portion of the injected macrophages is observed for at least 1 week [216]. Bromodeoxyuridine labeling demonstrated that Ly6C $\mathrm{C}^{\text {high }}$ monocytes take 2-3 days to fully accumulate in tumor tissue after arriving in the circulation [217].

Due to their well-known ability to kill tumor cells, their antigenpresenting properties and their accumulation in necrotic/hypoxic regions of solid tumors, classically activated (by IFN- $\gamma$ and/or LPS) macrophages were explored as cancer treatment already in the 1990s $[110,218,219]$. Proper macrophage culture upscaling techniques were even developed to facilitate their clinical use in humans [218]. Indeed, the natural tendency of macrophages to be attracted to hypoxic regions in tumors presented an opportunity to target these hard-to-access areas frequently associated with resistance to chemo- and radiotherapy [220,221]. Moreover, injected macrophages had previously been shown to enter small lung metastases of B16 melanoma [222]. More recently, intraperitoneal injected macrophages were found to traffic into an experimentally-induced inflamed pancreas, indicating their potential use in a broad range of inflammation-associated pathologies [223]. The latter experiment also indirectly demonstrated that organs in the abdominal cavity are likely to be targeted with much higher efficiency after intraperitoneal injection compared to systemic injection [223]. However, since similar inflammation site-targeting efficiencies can be achieved with macrophage cell lines such as the mouse RAW264.7 as opposed to primary macrophages, a rather non-selective accumulation is to be suspected [224].

In the context of gene therapy, macrophages can be transduced with viral vectors coding for prodrug-activating enzymes. Additional layers of selectivity can be obtained by incorporating hypoxia response elements (HRE), driven by transcription factors such as hypoxia-inducible factors [225,226]. The in vivo validation of obtaining increased selectivity by combining HRE with therapeutic genes stalled about 20 years ago, but is still worth exploring since the clinical efficacy of systemic macrophage-based cell therapies turned out to be limited, despite being well-tolerated [218,227]. The potential of multi-layered selectivity using tumor-homing macrophages as vehicles has been described for a hypoxia-regulated oncolytic adenovirus, whose proliferation is additionally restricted to prostate tumor cells by prostate-specific promoter elements [228]. When the criteria are met, local amplification of the viral agents can then compensate for the low number of transduced cells that were able to reach the target site [229]. Additional therapeutic efficiency can be obtained when cell-delivered oncolytic virus therapy is initiated directly after tumor radiation therapy 
[230,231]. Unfortunately, no data on the biodistribution of these vectors in organs other than the prostate tumor were included [228]. Of note, systemically administered oncolytic viruses are impeded by the same barriers as nanoparticles, e.g. interaction with blood cells, compromised access to non-vascularized regions within the tumor, dense collagen deposition and elevated interstitial pressure [232].

Summarized, much is known about ex vivo culture of primary monocytes. Employing this versatile cell type as drug delivery vehicles is tempting because they are involved in many pathologies and are welltolerated after administration. Furthermore, the ability of monocytes to traffic deep into badly perfused tumors makes them appealing in these settings as well. However, monocytes are hardwired to react heavily to foreign substances and can therefore be difficult to manipulate for therapeutic purposes.

\subsection{Myeloid-derived suppressor cells}

Tumor-associated soluble factors induce and release immature immune cells of myeloid lineage from the bone marrow. These cells cause immunosuppression in the TME and are therefore named myeloid-derived suppressor cells (MDSCs) [233,234]. They constitute a heterogeneous population that can be broadly subdivided into polymorphonuclear (PMN-MDSC) and monocytic (M-MDSC) subsets. However, solely based on immunophenotypic markers, MDSCs are difficult to distinguish from their more mature counterparts and therefore require functional evaluation of their immature and immunosuppressive nature $[233,235,236]$. Interpretation of the already vague distinction between MDSC and mature myeloid cells is further complicated by non-standardized methods of MDSC isolation and storage [237,238]. Furthermore, MDSCs can differentiate into mature myeloid cells and vice versa within the TME [239]. Nevertheless, atypical myeloid subsets with distinct properties such as decreased cellular density have been clearly identified in cancer patients [240].

In tumor-bearing mice, a highly increased number of mostly PMNMDSCs are mobilized from the bone marrow and accumulate in organs such as the lungs, the liver and the spleen [241]. The latter represents an important organ in myeloid cell dissemination and even forms a major site of extramedullary hematopoiesis in some cancer models. Splenectomy delays tumor growth by reducing aberrant myeloid cell responses in the blood and tumor tissues [242,243]. Adoptively transferred MDSCs traversed the lung and liver capillary networks more rapidly in tumor-bearing mice than in healthy control mice and subsequently massively accumulated in the spleen [241]. In addition, tumor-bearing mice supported MDSC survival, decreased their susceptibility to apoptosis and stimulated their proliferation in the spleen [241]. Hypothetically, tumor-associated factors inhibit the expression of apoptotic markers on adoptively transferred MDSCs and consequently prevent their sequestration in organs associated with cell clearance.

The increased production, survival and mobilization of MDSCs results in more circulating and recruited cells into the tumor via a multitude of chemokines, cytokines and mediators of inflammation [244]. Interestingly, increased blood PMN-MDSCs do not always correlate with increased numbers in the TME due to impaired migration functions resulting from e.g. downregulation of chemokine receptors CXCR1 and CXCR2 [240]. Conversely, the highly CXCR2-dependent recruitment of PMN-MDSCs into the TME can be blocked either by monoclonal antibodies [245] or by small molecule inhibitors [246]. Utilizing such inhibitors leads to improved therapeutic outcomes when combined with anti-PD-1 treatment, anti-CTLA-4 treatment or adoptive transfer of T cells [246,247].

Bone marrow-derived MDSCs can be generated in vitro via GM-CSF and IL-6 supplementation to standard culture medium [248]. This method was used to investigate the biodistribution of adoptively transferred PMN-MDSCs in an orthotopic PyMT-MMTV mammary tumor mouse model [249]. Intravenously injected DiD-labeled PMN-
MDSCs mostly accumulated in the liver, the spleen and the lungs but fluorescence was also observed at the primary tumor, both at 48 hours and at 7 days after injection [249]. Moreover, injected MDSCs also colocalized with tumor metastases in the adrenal gland but off-target splenic accumulation was seen as well [249]. However, histological verification should be performed to evaluate whether this acquired fluorescence originated from intact labeled MDSCs [249,250].

The tumor homing tendency of mouse $\mathrm{Ly} 6 \mathrm{C}^{+}$MDSCs has been exploited to more efficiently deliver oncolytic viruses into the TME [251]. However, this study used an intrahepatic tumor model in which the obtained "tumor-specific" MDSCs are likely confounded with MDSCs residing in the liver. Another study reported delivery of attenuated Listeria monocytogenes bacteria engineered to express tumor antigens via MDSCs into mouse primary tumors and metastases [252]. In addition to their ability to elicit antigenic responses, these bacteria infect and kill tumor cells as well [253]. Despite infecting both MDSC populations, bacterial proliferation and subsequent delivery of $L$. monocytogenes occurred mostly via M-MDSCs and resulted in equally efficient delivery at the primary tumor and tissue metastases of both young and old mice. In contrast, a much lower bacterial load was demonstrated in the spleen [252] although assessing the contribution of the MDSC as vehicles is difficult since much of the selectivity might be related to the bacteria alone.

In conclusion, it remains unclear whether MDSCs demonstrate higher tumor recruitment potential compared to mature myeloid cells. Further studies are needed to answer this question and to unravel how the migration of MDSCs differs from other immune cell populations.

\section{8. $T$ cells}

The trafficking properties of $\mathrm{T}$ cells are currently intensely investigated due to their potent capacity to eradicate tumor cells, certainly since the availability of clinically applicable methods to genetically engineer CTLs that are able to kill cells expressing an antigen of choice [254,255]. Some of the specific properties of lymphocytes in relation to myeloid cell trafficking will be summarized (Table 1), for more detailed information readers are kindly referred to the excellent up-to-date reviews on this subject [62,256-259].

Cells of the adaptive immune system show some remarkable differences with innate immune cells. T cells develop in the thymus instead of the bone marrow, enter the circulation in a "naive" state and require multistep activation to initiate their effector functions. Moreover, they have the capacity to proliferate upon activation thus amplifying their immunological contribution and to produce longlasting memory cells. Importantly, this property is exploited to acquire sufficient cells for $\mathrm{T}$ cell-based therapies [260]. In further contrast to myeloid cells, naive $T$ cells mainly traffic through lymphoid tissues via L-selectin and CCR7-mediated interactions with corresponding ligands i.e. PNAd and CCL19/21, respectively, on specialized high endothelial venules in lymph nodes [261]. Once activated in these lymphoid tissues, T lymphocytes downregulate their surface expression of CCR7, egress the lymph node via their sphingosine 1 phosphate receptor 1 and upregulate other adhesion receptors to acquire the capacity to migrate into non-lymphoid tissues [56,62]. Interestingly, these newly expressed adhesion receptors partly differ depending on the tissue location of the priming lymph node and antigen-presenting dendritic cells, thereby bestowing a certain vascular "ZIP code" on the activated T lymphocyte. This combination of acquired adhesion receptors/ligands corresponds to adhesion ligands/receptors in the tissue where the priming occurred $[62,256]$. Consequently, eliciting adaptive immunity through vaccination preferentially occurs in the targeted tissue. Some homing receptors/ligands such as CXCR3 and PSGL-1 are upregulated independent of the activation site and inflammation utilizes many of the homing receptors/ligands normally restricted to specific tissues in steady state conditions [256]. This inflammation-induced upregulation of chemokines and vascular receptors is often actively inhibited (e.g. by 
VEGF) in solid tumors, but can be reinitiated by experimentally introducing stimulators of inflammation such as Toll-like receptor (TLR)agonists [256,262,263].

Aberrant tumor blood vessel morphology also contributes to defective $\mathrm{T}$ cell homing. In this regard, low-dose anti-angiogenic therapy might be more beneficial in treating solid tumors than high-dose therapies as the former allows normalization of the tumor vasculature which allow more efficient recruitment, adhesion and extravasation of $\mathrm{T}$ cells [48]. In addition to normalizing the tumor vasculature, tumormatching chemokine receptors such as CXCR2 [264], CX3CR1 [265], CCR4 [266] and CCR2 can all be genetically introduced into T cells [257,267,268]. Unfortunately, clinical studies with therapeutic T cells have indicated that addition of these chemokine receptors only modestly improved the infiltration in humans [257]. Yet, an interesting link between myeloid cells and lymphocyte recruitment was recently demonstrated: infiltration of immunosuppressive PMN-MDSCs in mouse tumors was substantially reduced upon administration of a small molecule inhibitor of both CXCR1 and CXCR2 [246]. In turn, this greatly enhanced $\mathrm{T}$ cell accumulation in these tumors, indicating that myeloid cells residing in the TME contribute to constraining lymphocyte recruitment.

Chimeric antigen receptors (CARs) were originally engineered to confer selective T cell-mediated killing of target cells bearing the antigen of choice independent of MHC. However, the same principle can be used to elicit expression of a certain gene of choice after recognition of the antigen. In essence, this makes CAR T cells drug delivery vehicles for therapeutic genes such as cytokines and they are therefore termed "T cells redirected for universal cytokine killing" (TRUCKs) [269]. For example, TRUCKs can be used to locally express IL-12 after recognition of its cognate antigen [270]. Indeed, this is a very elegant system because IL-12 is a cytokine that elicits potent Th1 cellular immune responses but cannot be administered systemically due to unacceptable toxicity [271]. Its expression can be linked to either CAR or TCR activation through incorporation of a nuclear-factor of activated $\mathrm{T}$ cells (NFAT) minimal promoter [269]. Due to the proliferative property of T cells, TRUCKs which constitutively express IL-12 can also be constructed [272]. Systemic injection of a small number of such IL-12 TRUCKs will not elicit toxic effects [273]. However, after encountering the targeted cells, $\mathrm{T}$ cells massively proliferate and exponentially increase local IL-12 concentrations until therapeutic effects are achieved [272]. Nevertheless, induced expression of IL-12 is considered more safe than constitutively expressed IL-12 [269]. Cytotoxic T cells can also be used to deliver drugs adsorbed to their surface. For example, Jones et al. (2016) conjugated drug-loaded lipid nanoparticles to the surface of T cells [274]. Upon recognition of their cognate antigen, the $\mathrm{T}$ cells secrete perforins which not only lyse the target cells but also release the drug from the adsorbed lipid nanoparticles. The drawback of CAR or TCR-mediated release is that it requires prior knowledge on the targeted antigen. Choosing the wrong antigen can result in severe "ontarget off-tumor" side effects. Moreover, targeting a single antigen is often insufficient in constantly evolving heterogeneous pathologies such as cancer [56,256]. Alternatively, more stable TME-associated antigens such as VEGFR-2 can be targeted as well [275].

The development of multiple generations of CAR T cells have provided extensive insight into the possibilities of genetic engineering. In fact, CARs have also been inserted in other cell types [276]. Facilitated by gene-editing technology, researchers are exploring genetic knockout of MHC-associated genes to prevent host immune recognition of adoptively transferred cells $[277,278]$. A complementary strategy is to remove native $\mathrm{T}$ cell receptors from CAR T cells to prevent graft versus host reactions. Consequently, allogeneic cells can be used to create "off the shelf" cell delivery vehicles.

\section{Natural killer cells}

Treating tumors with bioengineered CAR T cells requires sufficient functional $\mathrm{T}$ cells to reach the tumor. This poses a problem in treating immunosuppressive tumors or "cold tumors", in which T cells cannot permeate [279]. Natural killer (NK) cells are currently explored to overcome some of the limitations associated with T cells [280]. TCR/ CD3-negative NK cells are lymphocytes which possess essential functions for the innate immune system. This makes NK cells interesting for cancer immunotherapy [281]. Their cytotoxic effector mechanisms include perforin/granzyme-containing granules, antibody-dependent cellmediated cytotoxicity (via Fc $\gamma$ RIII or CD16) and cell death through death-receptor and interferon- $\gamma$ mediated pathways [282]. Through both activating and inhibitory receptors, they can recognize target cells based on reduced expression of inhibitory ligands and/or increased expression of activating ligands and thus exert broad and rapid cytotoxicity against e.g. virus-infected cells or tumor cells [283]. For example, loss of MHC class I molecules (inhibitory signal) and/or increased expression of stress ligands such as MICA, MICB and ULBPs (activating signal) makes cells susceptible to NK cell-mediated lysis [283].

Fueled by the success of CAR T cells, NK cells are now also genetically modified with CARs [284]. In essence, this makes CAR-NK cells function as adaptive immune cells via their tumor antigen-recognizing CAR and as innate immune cells through their native receptors. This combination makes tumor escape through downregulation of CAR targets less likely. Moreover, no cytokine release syndrome is expected since NK cells do not proliferate upon stimulation [285]. Importantly, because NK cell-mediated cytotoxicity is MHC-independent, they lack the potential to cause graft-versus-host disease, which allows NK cells to be used as off-the-shelf allogeneic therapeutics [283].

Whereas all lymphoid and non-lymphoid tissues contain NK cells, most human NK cells in the lymph nodes are of the immature

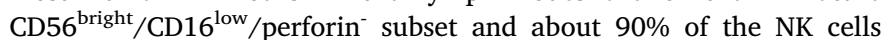
found in the blood and the spleen are of the more mature cytolytic $\mathrm{CD} 6^{\mathrm{dim}} / \mathrm{CD} 16^{+} /$perforin $^{+}$subset(Table 2) [282,286]. The non-cytotoxic CD56 $6^{\text {bright }}$ and cytotoxic CD56 ${ }^{\text {dim }}$ NK cell subsets are also characterized by a different set of chemokine receptors [287,288]. In mice, the functional classification occurs through CD27/CD11b characterization. Immature mouse NK cells are double negative CD27-/CD11bNext, they mature into CD27+/CD11b- to CD27+/CD11b + and finally CD27-/CD11b + NK cell subsets [289].

In vitro cultivation of primary NK cells is technically challenging. Current strategies therefore rely on infusing irradiated NK cells from the human NK-92 cell line which retain their cytotoxicity but exhibit suppressed proliferation to prevent their permanent engraftment [283]. As a result, CAR-NK-92 cells remain in circulation for about 48 hours [290] and are no longer detectable within 7 days of adoptive infusion (Table 1) [291]. Alternatively, human embryonic stem cells [292] or CAR-NK cells derived from induced pluripotent stem cells demonstrate equal anti-tumor capacities as CAR T cells, but with a better safety profile [293,294]. Of note, activating the NK cells prior to adoptive transfer significantly improves their tumor trafficking in mice 1 hour after administration but also causes them to accumulate in off-target organs such as the lungs, the liver and the spleen [282]. Such activated NK cells also demonstrate accumulation at pulmonary metastases. This interesting property of NK cells was used to deliver membrane-linked doxorubicin to B16 melanoma metastases in the lung thereby severely concentrating doxorubicin at these sites [295].

\section{Employing nanoparticles to load tumor-targeting immune cells}

The focus of cell-based drug delivery is gradually merging with the field of nanoparticles [296]. Careful nanoparticle design considerably improves drug pharmacokinetics, but still lacks efficient targeting capacity. However, when combined with the inherent properties of immune cells to target inflamed regions, a marked increase in delivery 
efficiency has been reported [148]. For example, in a recent study Ly6C ${ }^{\text {high }}$ monocytes loaded with paclitaxel-containing $\mathrm{pH}$-sensitive micelles demonstrated increased paclitaxel accumulation in the primary tumor and pulmonary metastases when compared to micelles directly injected in the blood [297]. Moreover, about one decade ago macrophages loaded with nanoparticles containing an antiviral drug severely prolonged drug release and brain deposit in an HIV-1 encephalitis mouse model [298]. However, ex vivo culture of monocytes and loading them with nanoparticles or reporter genes can negatively impact the delivery efficiency of these cells [299].

Another example is to coat nanoparticles with anti-CD11b to target circulating myeloid cells. After inducing an acute inflammation reaction in the TME by photosensitization therapy, neutrophils that have taken up these anti-CD11b nanoparticles are recruited into the TME [300]. Moreover, since neutrophil infiltration has been observed after radiation therapy it can complement this frequently used cancer treatment modality [301]. Although this strategy resulted in higher delivery of nanoparticles in the TME, radiation therapy still requires knowledge about the exact position of the TME and hence, is not practical for targeting metastases of unknown locations. Nevertheless, systemic immunotherapy using antibodies directed against melanoma antigens is also associated with neutrophil influx and could therefore benefit from this approach as well [302]. Still, two additional problems arise when employing immune cells to deliver therapeutic nanoparticles on site: [1] drug leakage from the nanoparticle into the immune cell can compromise its function and viability, [2] immune cell recruitment inevitably results in excessive drug deposition into sequestration organs such as the lungs, the liver and the spleen [303].

For example, in a recent study Ly6 $\mathrm{C}^{\text {high }}$ monocytes loaded with paclitaxel-containing $\mathrm{pH}$-sensitive micelles demonstrated increased paclitaxel accumulation in the primary tumor and pulmonary metastases when compared to micelles directly injected in the blood [297]. Moreover, about one decade ago macrophages loaded with nanoparticles containing an antiviral drug severely prolonged drug release and brain deposit in an HIV-1 encephalitis mouse model [298]. However, ex vivo culture of monocytes and loading them with nanoparticles or reporter genes can negatively impact the delivery efficiency of these cells [299].

Multiple strategies have been described to load cells with synthetic or biological therapeutic agents. For example, enzymes can be encapsulated into red blood cells via hypotonic dialysis and isotonic resealing [304] or can be attached to cell membranes via integration of biotin-phospholipid integration followed by binding of avidin-conjugated agents [305]. Alternatively, cell-penetrating peptides are used to facilitate intracellular delivery of hydrophilic agents or macromolecular substances [306]. Of particular interest is the use of nanoparticle drug carriers which can be coated with cell membranes $[307,308]$ or attached/loaded into the cellular vehicles. Membrane coating transfers beneficial properties of the source cells (e.g. targeting ligands/receptors and "don't-eat-me" signals) to the used nanoparticles. This strategy is covered in the review by Zhang et al. (2019) [309]. Attaching nanoparticles to endogenous circulating cells is known as "cellular hitchhiking" [310] and can be achieved by non-covalent adsorption via hydrophobic, electrostatic, Van der Waals or hydrogen bonding [310,311]. In addition, drug carriers can be attached to cells via receptor-ligand interactions [312] or they can even be covalently attached to functional thiol [313] or amine [314] residues on the cell surface [315]. Internalization of adherent nanoparticles through endocytosis, pinocytosis or phagocytosis is considered a limiting factor but can also be used to load therapeutic cargo in phagocytes [315,316]. However, the latter approach requires proper protection against intracellular degradation mechanisms.

\section{Challenges and future perspectives}

\subsection{The lungs, the liver and the spleen}

To scout the entire organism for possible target sites such as cancer metastases, systemic administration is mandatory, but also obligates the injected cells to pass through certain checkpoints. Typically, the lungs are the first checkpoint encountered after intravenous injection [303]. Priming or activation of immune cells leads to cytoskeleton rearrangements which makes them more rigid and exhibit a higher tendency to get stuck into capillary beds such as in the lungs $[159,165,166,317]$. Likewise, a clear size-dependent pulmonary accumulation of intravenously injected nanoparticles has been described in rats $[318,319]$. Simultaneous treatment with vasodilation agents such as sodium nitroprusside helps cells to pass through the lungs more rapidly, further indicating that physical obstruction is at least partly responsible for the pulmonary retention of injected immune cells [320,321]. After their release from the lungs, systemically injected cells are distributed among all other organs.

The two main sites of subsequent sequestration are the liver and the spleen [303,322-324]. It is difficult to assess what exactly happens to the immune cells in these two organs and whether the observed retention in these secondary "checkpoints" is reversible. One possibility is that the immune cells accumulate for mere physical reasons (e.g. large vascular volume of these organs or small capillary diameters) [325]. Given that spleen contraction is susceptible to $\alpha$-adrenoceptor agonists, treatment with adrenaline or epinephrine can possibly elicit leukocyte mobilization from this reservoir [326]. Although spleen contraction substantially increases blood hematocrit in horses and dogs, it appears to be far less influential in humans [327]. Nonetheless, catecholaminemediated demargination combined with expulsion of splenic leukocytes likely contributes to increased circulation of adoptively transferred immune cells [157,158,326]. Moreover, immune cell mobilization can readily be enhanced through exercise, which increases immune cell infiltration in tumors and subsequent therapeutic effect [257]. Removing the spleen before injection of immune cells resulted in increased liver accumulation of these cells, but their short-term trafficking to tumors remained unaffected [328]. This key observation could indicate that the luminal surface of the tumor vasculature is saturated with endogenous immune cells, hindering injected cells to find an available attachment site [329]. Consequently, intratumoral cell accumulation is a gradual process that requires multiple days or even weeks to complete.

Another possibility is that injected immune cells are actively arrested by other cells. The removal of dead or compromised cells from circulation (efferocytosis) by cells of the MPS is a major function of both the spleen and the liver [330]. Any deviation of the cell surface caused by experimental manipulations such as isolation, ex vivo culture, transfection and labeling likely activates markers of efferocytosis. This is exemplified by the rapid disappearance of optimally stored red blood cells (RBCs) after transfusion [331]. In addition, other "eat me" signals such as phosphatidylserine externalization, changes in either charge or glycosylation patterns, ICAM-1 alterations and exposure of calreticulin on the cell surface all mark a cell for phagocytosis by the MPS [332]. Interestingly, masking phosphatidylserine exposure via annexin $\mathrm{V}$ prevented subsequent phagocytosis and could therefore be explored as a way to decrease the arrest of adoptively transferred immune cells in organs participating in efferocytosis [333]. Our group has recently demonstrated that depleting the MPS via pretreatment with clodronate liposomes drastically increased both the circulatory half-life and tissue accumulation efficiency of infused bone marrow-derived monocytes [329]. In addition, no change in monocyte distribution pattern was observed after depletion of endogenous neutrophils [329]. Alternative strategies could consist of depleting cells that express apoptotic markers before their infusion, or to incorporate cell surface "don't eat me" signals such as CD47 into the cellular vehicles [334]. In addition, adding 
anti-apoptotic agents to the culture medium can perhaps diminish the fraction of injected cells that express apoptotic markers [335]. Compared to RBCs, decorating the cell surface with polyethylene glycol (PEGylation) is likely less efficacious on immune cells as these modifications have been shown to interfere with surface molecules. Hence, PEGylation will likely inhibit the migration cascade of immune cells [113].

\subsection{Prolonging the circulatory half-life of immune cells as the first pillar}

Despite the preference for recruiting certain immune cell types to the TME in the long-term, the short-term accumulation of injected immune cells is mainly of non-specific nature [303,328]. Retention of injected immune cells therefore likely depends primarily on physical properties such as tumor perfusion, total tumor blood volume and cell flow rate. This underscores the importance of optimizing essential physical properties such as the circulatory half-life rather than identifying optimal injection sites [336]. Nevertheless, the rapid recruitment of endogenous neutrophils in acute inflammatory contexts suggests that some level of short-term trafficking of specific immune cell subsets is possible but we should bear in mind the high number of circulating neutrophils in steady-state conditions [337] and the induced reactive neutrophilia in inflammatory conditions which further helps circulating neutrophils to accumulate at the pathological site by ensuring their constant passage [338].

Considering these general remarks, prolonging the circulatory halflife of cell vehicles should be designated as the first pillar in this research field and preventing the immediate recognition of altered cell surfaces should be prioritized in future research endeavors (Fig. 4). Even when injected cells are not immediately sequestered in organs such as lungs, spleen and liver, only a small portion of them will actually travel through the blood vessels of their targets sites. Moreover, interaction with the endothelium through rolling, adhesion and transmigration is only reserved for a minority of passing cells and thus, the majority of the cellular vehicles will return to the heart and the lungs to restart the cycle. Therefore, like passively flowing particles, these injected cells also largely depend on their circulatory half-life to increase their chances of successfully engaging the adhesion cascade at their target sites.

Based on the demargination and consequential leukocytosis mediated by glucocorticosteroid administration, it would perhaps be interesting to further investigate whether glucocorticosteroids can also increase the circulatory half-life of adoptively transferred immune cells [339-342]. Moreover, this class of anti-inflammatory and strongly immunomodulating drugs also prevents apoptosis of neutrophils and can potentially inhibit delayed adaptive immune responses against therapeutic transgenes [343]. Despite the existence of a vascular ZIPcode system for primed effector $\mathrm{T}$ cells, specific tissue migration is likely hampered by the shared expression of adhesion molecules in different organs [62]. Therefore, instead of trying to acquire increased specificity via addition of adhesion receptors in cellular vehicles, it would perhaps be more efficient to mask the non-specific receptors/ ligands already present on these cells. This would then substantially decrease interaction with adhesion receptors in non-targeted organs and possibly increase circulation half-life and target site accumulation of the injected cells.

One of the many exciting evolutions of the past decade is the merging of the nanoparticle delivery field with research on immune cell trafficking [148,300,302,344,345]. The main strategy is to bestow immune cell-targeting properties to nanoparticles containing a therapeutic agent. After systemic injection, circulating immune cells then adsorb or engulf the nanoparticles, effectively guiding them into diseased tissues such as solid tumors. Given that leakage from the nanoparticles can be constrained, "piggybacking" endogenous immune cells can potentially bypass the clearance problems associated with ex vivo manipulations.

\subsection{Conditional release as the second pillar}

Even if we assume that the hurdles of efferocytosis and circulatory half-life could be adequately addressed, unwanted off-target accumulation by physical means such as differences in total vascular volume between organs, will always hamper drug delivery efficiency. By taking advantage of the many possibilities that cells offer for genetically engineering multilayered selectivity, investing substantial research efforts into developing tools for conditional release should form the second pillar of obtaining true targeted delivery (Fig. 4). Currently pursued

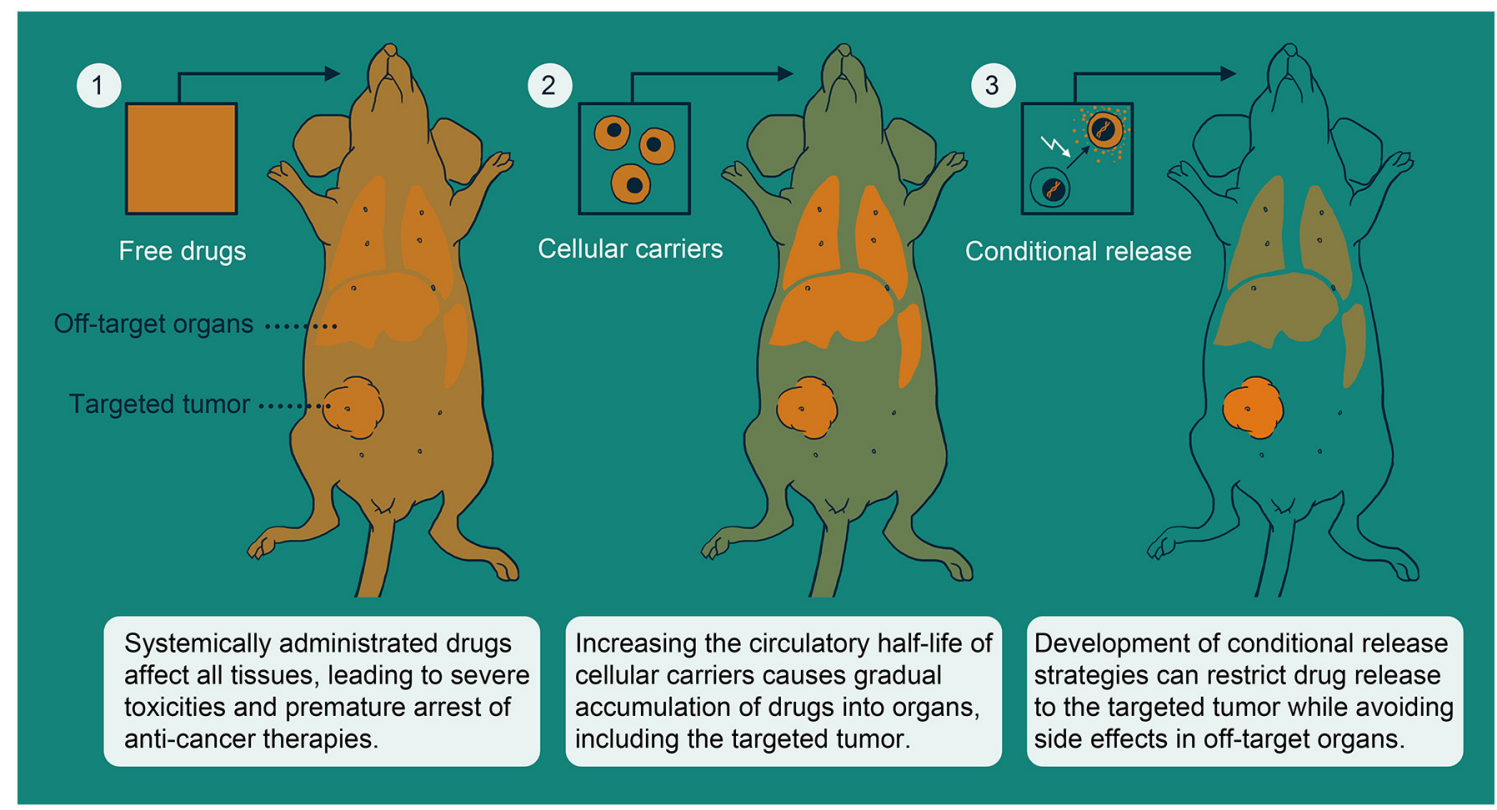

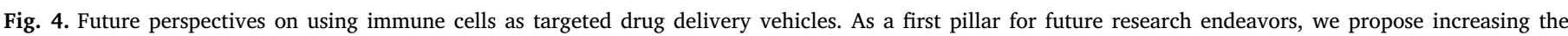

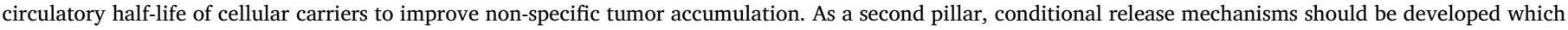
release the drug from its carrier based on specific cues in the tumor. 
avenues include local amplification of adoptively transferred $\mathrm{T}$ cells after recognition of their cognate antigen [346], local amplification of oncolytic viruses [347] and development of genetic switches [348-350] that control transgene expression based on environmental cues. In our opinion, the development of multilayered genetic switches has the most potential in terms of flexibility (i.e. back-and-forth optimization and adaptation). These switches do not necessarily require prior knowledge about a specific antigen, but can link cell phenotypic changes based on environmental cues in the TME with the conditional expression of a therapeutic transgene. For example, combining a miRNA-driven approach [351] with other selective expression techniques such as polarization-dependent promoters [352] or enhancers [353] can undoubtedly ensure specific transgene expression in targeted tissues. In this regard, integrating hypoxia responsive elements (HREs) in the genetic constructs can confine transgene expression to the badly perfused tumor regions [225,226]. Nevertheless, these HREs could also impose too much restrictions (e.g. early metastatic sites do not necessarily contain hypoxic regions) and therefore limit the therapeutic relevance of this construct.

\section{Acknowledgements}

The authors acknowledge funding from the Research Foundation Flanders (1119318N, G087516N) and the Special Research Fund from Ghent University (BOF15/GOA/013).

\section{References}

[1] A. Skuse, Wellcome Trust-Funded Monographs and Book Chapters. Constructions of Cancer in Early Modern England: Ravenous Natures, Palgrave Macmillan (c) Alanna Skuse 2015, Basingstoke (UK), 2015.

[2] W. Zhang, P. Huang, Cancer-stromal interactions: role in cell survival, metabolism and drug sensitivity, Cancer Biol. Ther. 11 (2) (2011) 150-156.

[3] M. Arruebo, et al., Assessment of the evolution of cancer treatment therapies, Cancers 3 (3) (2011) 3279-3330.

[4] S.A. Abraham, et al., The liposomal formulation of doxorubicin, Methods Enzymol. 391 (2005) 71-97.

[5] G. Kohler, C. Milstein, Continuous cultures of fused cells secreting antibody of predefined specificity, Nature 256 (5517) (1975) 495-497.

[6] Y.H. Yun, B.K. Lee, K. Park, Controlled drug delivery: historical perspective for the next generation, J. Control. Release 219 (2015) 2-7.

[7] C.N. Simonti, J.A. Capra, The evolution of the human genome, Curr. Opin. Genet. Dev. 35 (2015) 9-15.

[8] D.S. Krause, R.A. Van Etten, Tyrosine kinases as targets for cancer therapy, N. Engl. J. Med. 353 (2) (2005) 172-187.

[9] J. Sleeman, P.S. Steeg, Cancer metastasis as a therapeutic target, Eur. J. Cancer (Oxford, England: 1990) 46 (7) (2010) 1177-1180.

[10] S.A. Eccles, D.R. Welch, Metastasis: recent discoveries and novel treatment strategies, Lancet (London, England) 369 (9574) (2007) 1742-1757.

[11] K.W. Hunter, R. Amin, S. Deasy, N.H. Ha, L. Wakefield, Genetic insights into the morass of metastatic heterogeneity, Nat. Rev. Cancer 18 (4) (2018) 211-223.

[12] B. Meibohm, H. Derendorf, Basic concepts of pharmacokinetic/pharmacodynamic (PK/PD) modelling, Int. J. Clin. Pharmacol. Ther. 35 (10) (1997) 401-413.

[13] H. Derendorf, B. Meibohm, Modeling of pharmacokinetic/pharmacodynamic (PK/ PD) relationships: concepts and perspectives, Pharm. Res. 16 (2) (1999) 176-185.

[14] L. Rossi, et al., Engineering erythrocytes for the modulation of drugs' and contrasting agents' pharmacokinetics and biodistribution, Adv. Drug Deliv. Rev. 106 (Pt A) (2016) 73-87.

[15] H.H. Wong, S. Halford, Dose-limiting toxicity and maximum tolerated dose: still fit for purpose? Lancet Oncol. 16 (13) (2015) 1287-1288.

[16] H.G. Stampfer, G.M. Gabb, S.B. Dimmitt, Why Maximum Tolerated Dose? (2019).

[17] C. Keywood, Chapter 17 - Clinical development: present and future, in: R.G. Hill, H.P. Rang (Eds.), Drug Discovery and Development, Second edition, Churchill Livingstone, 2013, pp. 239-258.

[18] K. Venkatakrishnan, et al., Optimizing oncology therapeutics through quantitative translational and clinical pharmacology: challenges and opportunities, Clin. Pharmacol. Ther. 97 (1) (2015) 37-54.

[19] J. Zugazagoitia, et al., Current challenges in cancer treatment, Clin. Ther. 38 (7) (2016) 1551-1566.

[20] M. Pein, T. Oskarsson, Microenvironment in metastasis: roadblocks and supportive niches, Am. J. Physiol. Cell. Physiol. 309 (10) (2015) C627-C638.

[21] J. Brabek, D. Rosel, M. Fernandes, Pragmatic medicine in solid cancer: a translational alternative to precision medicine, OncoTargets Ther. 9 (2016) 1839-1855.

[22] S. Maman, I.P. Witz, A history of exploring cancer in context, Nat. Rev. Cancer 18 (6) (2018) 359-376.

[23] P. Vaupel, L. Harrison, Tumor hypoxia: causative factors, compensatory mechanisms, and cellular response, Oncologist 9 (Suppl. 5) (2004) 4-9.
[24] P.M. Gullino, S.H. Clark, F.H. Grantham, The interstitial fluid of solid tumors, Cancer Res. 24 (1964) 780-794.

[25] Y. Kato, et al., Acidic extracellular microenvironment and cancer, Cancer Cell Int 13 (1) (2013) 89.

[26] H. Maeda, H. Nakamura, J. Fang, The EPR effect for macromolecular drug delivery to solid tumors: improvement of tumor uptake, lowering of systemic toxicity, and distinct tumor imaging in vivo, Adv. Drug Deliv. Rev. 65 (1) (2013) 71-79.

[27] B. Warren, Tumor blood circulation: angiogenesis, vascular morphology and blood flow of experimental and human tumors, The Vascular Morphology of Tumors, CRC Press, Inc., Florida, 1979, pp. 1-47.

[28] D.J. Chaplin, P.L. Olive, R.E. Durand, Intermittent blood flow in a murine tumor: radiobiological effects, Cancer Res. 47 (2) (1987) 597-601.

[29] T.P. Padera, E.F.J. Meijer, L.L. Munn, The lymphatic system in disease processes and cancer progression, Annu. Rev. Biomed. Eng. 18 (2016) 125-158.

[30] C.C. DuFort, M.J. Paszek, V.M. Weaver, Balancing forces: architectural control of mechanotransduction, Nat. Rev. Mol. Cell. Biol. 12 (5) (2011) 308-319.

[31] R. Xu, A. Boudreau, M.J. Bissell, Tissue architecture and function: dynamic reciprocity via extra- and intra-cellular matrices, Cancer Metastasis Rev. 28 (1-2) (2009) 167-176.

[32] C.H. Heldin, K. Rubin, K. Pietras, A. Ostman, High interstitial fluid pressure - an obstacle in cancer therapy, Nat. Rev. Cancer 4 (10) (2004) 806-813.

[33] M.A. Swartz, A.W. Lund, Lymphatic and interstitial flow in the tumour microenvironment: linking mechanobiology with immunity, Nat. Rev. Cancer 12 (2012) 210.

[34] Y. Matsumura, H. Maeda, A new concept for macromolecular therapeutics in cancer chemotherapy: mechanism of tumoritropic accumulation of proteins and the antitumor agent smancs, Cancer Res. 46 (12 Pt 1) (1986) 6387-6392.

[35] H. Maeda, Tumor-selective delivery of macromolecular drugs via the EPR effect: background and future prospects, Bioconjug. Chem. 21 (5) (2010) 797-802.

[36] F. Danhier, To exploit the tumor microenvironment: since the EPR effect fails in the clinic, what is the future of nanomedicine? J. Control. Release 244 (2016) $108-121$.

[37] M. Yu, J. Zheng, Clearance pathways and tumor targeting of imaging nanoparticles, ACS Nano 9 (7) (2015) 6655-6674.

[38] S.K. Hobbs, et al., Regulation of transport pathways in tumor vessels: role of tumor type and microenvironment, Proc. Natl. Acad. Sci. U. S. A. 95 (8) (1998) 4607-4612.

[39] J.S. Suk, Q. Xu, N. Kim, J. Hanes, L.M. Ensign, PEGylation as a strategy for improving nanoparticle-based drug and gene delivery, Adv. Drug Deliv. Rev. 99 (Pt A) (2016) 28-51.

[40] Q. Yang, S.K. Lai, Anti-PEG immunity: emergence, characteristics, and unaddressed questions, Wiley Interdiscip. Rev. Nanomed. Nanobiotechnol. 7 (5) (2015) 655-677.

[41] Y. Bi, et al., Actively targeted nanoparticles for drug delivery to tumor, Curr. Drug Metab. 17 (8) (2016) 763-782.

[42] J.W. Nichols, Y.H. Bae, EPR: evidence and fallacy, J. Control. Release 190 (2014) 451-464.

[43] K. Park, The beginning of the end of the nanomedicine hype, J. Control. Release 305 (2019) 221-222.

[44] K.F. Pirollo, E.H. Chang, Does a targeting ligand influence nanoparticle tumor localization or uptake? Trends Biotechnol. 26 (10) (2008) 552-558.

[45] S. Wilhelm, et al., Analysis of nanoparticle delivery to tumours, Nat. Rev. Mater. 1 (2016) 16014.

[46] G. Baronzio, G. Parmar, M. Baronzio, Overview of methods for overcoming hindrance to drug delivery to tumors, with special attention to tumor interstitial fluid, Front. Oncol. 5 (2015) 165.

[47] W. Jiang, Y. Huang, Y. An, B.Y. Kim, Remodeling tumor vasculature to enhance delivery of intermediate-sized nanoparticles, ACS Nano 9 (9) (2015) 8689-8696.

[48] R.K. Jain, Normalization of tumor vasculature: an emerging concept in antiangiogenic therapy, Science (New York, N.Y.) 307 (5706) (2005) 58-62.

[49] J.S. Park, et al., Normalization of tumor vessels by Tie2 activation and Ang2 inhibition enhances drug delivery and produces a favorable tumor microenvironment, Cancer Cell 30 (6) (2016) 953-967.

[50] A.R. Cantelmo, et al., Inhibition of the glycolytic activator PFKFB3 in endothelium induces tumor vessel normalization, impairs metastasis, and improves chemotherapy, Cancer Cell 30 (6) (2016) 968-985.

[51] S. Sindhwani, et al., The entry of nanoparticles into solid tumours, Nat. Mater. 19 (5) (2020) 566-575.

[52] S. Pandit, D. Dutta, S. Nie, Active transcytosis and new opportunities for cancer nanomedicine, Nat. Mater. 19 (5) (2020) 478-480.

[53] Y. Yuan, Spatial heterogeneity in the tumor microenvironment, Cold Spring Harb. Perspect. Med. 6 (8) (2016) a026583.

[54] C. Murdoch, A. Giannoudis, C.E. Lewis, Mechanisms regulating the recruitment of macrophages into hypoxic areas of tumors and other ischemic tissues, Blood 104 (8) (2004) 2224-2234.

[55] J. Goc, W.H. Fridman, C. Sautes-Fridman, M.C. Dieu-Nosjean, Characteristics of tertiary lymphoid structures in primary cancers, Oncoimmunology 2 (12) (2013) e26836.

[56] L.L. van der Woude, M.A.J. Gorris, A. Halilovic, C.G. Figdor, I.J.M. de Vries, Migrating into the tumor: a roadmap for T cells, Trends Cancer 3 (11) (2017) $797-808$.

[57] J.N. Kather, et al., Topography of cancer-associated immune cells in human solid tumors, eLife 7 (2018) e36967.

[58] D. Vestweber, How leukocytes cross the vascular endothelium, Nat. Rev. Immunol. 15 (11) (2015) 692-704.

[59] M. Yang, D. McKay, J.W. Pollard, C.E. Lewis, Diverse functions of macrophages in 
different tumor microenvironments, Cancer Res. 78 (19) (2018) 5492.

[60] H.F. Dvorak, Tumors: wounds that do not heal. Similarities between tumor stroma generation and wound healing, N. Engl. J. Med. 315 (26) (1986) 1650-1659.

[61] E. Elinav, et al., Inflammation-induced cancer: crosstalk between tumours, immune cells and microorganisms, Nat. Rev. Cancer 13 (11) (2013) 759-771.

[62] R. Sackstein, T. Schatton, S.R. Barthel, T-lymphocyte homing: an underappreciated yet critical hurdle for successful cancer immunotherapy, Lab. Investig. 97 (2017) 669.

[63] A.D. Luster, R. Alon, U.H. von Andrian, Immune cell migration in inflammation: present and future therapeutic targets, Nat. Immunol. 6 (12) (2005) 1182-1190.

[64] W.A. Muller, Transendothelial migration: unifying principles from the endothelial perspective, Immunol. Rev. 273 (1) (2016) 61-75.

[65] T.R. Lee, et al., On the near-wall accumulation of injectable particles in the microcirculation: smaller is not better, Sci. Rep. 3 (2013) 2079.

[66] M.P. Bevilacqua, R.M. Nelson, Selectins, J. Clin. Invest. 91 (2) (1993) 379-387.

[67] S. Weinbaum, J.M. Tarbell, E.R. Damiano, The structure and function of the endothelial glycocalyx layer, Annu. Rev. Biomed. Eng. 9 (2007) 121-167.

[68] T. Yago, et al., Catch bonds govern adhesion through L-selectin at threshold shear, J. Cell Biol. 166 (6) (2004) 913-923.

[69] J. Kappelmayer, B. Nagy Jr., The interaction of selectins and PSGL-1 as a key component in thrombus formation and cancer progression, Biomed. Res. Int. 2017 (2017) 6138145.

[70] R.P. McEver, C. Zhu, Rolling cell adhesion, Annu. Rev. Cell Dev. Biol. 26 (2010) 363-396.

[71] E. Crockett-Torabi, Selectins and mechanisms of signal transduction, J. Leukoc. Biol. 63 (1) (1998) 1-14.

[72] R.P. McEver, Selectins: initiators of leucocyte adhesion and signalling at the vascular wall, Cardiovasc. Res. 107 (3) (2015) 331-339.

[73] S.C. Fagerholm, C. Guenther, M. Llort Asens, T. Savinko, L.M. Uotila, Beta2-integrins and interacting proteins in leukocyte trafficking, immune suppression, and immunodeficiency disease, Front. Immunol. 10 (2019) 254.

[74] I.D. Campbell, M.J. Humphries, Integrin structure, activation, and interactions, Cold Spring Harb. Perspect. Biol. 3 (3) (2011).

[75] H. Harjunpää, M. Llort Asens, C. Guenther, S.C. Fagerholm, Cell adhesion molecules and their roles and regulation in the immune and tumor microenvironment, Front. Immunol. 10 (2019) 1078.

[76] J. Li, et al., Conformational Equilibria and Intrinsic Affinities Define Integrin Activation, 36(5) (2017), pp. 629-645.

[77] M. Shimaoka, J. Takagi, T.A. Springer, Conformational regulation of integrin structure and function, Annu. Rev. Biophys. Biomol. Struct. 31 (2002) 485-516.

[78] C. Berlin, et al., $\alpha 4$ integrins mediate lymphocyte attachment and rolling under physiologic flow, Cell 80 (3) (1995) 413-422.

[79] T. Yago, N. Zhang, L. Zhao, C.S. Abrams, R.P. McEver, Selectins and Chemokines Use Shared and Distinct Signals to Activate beta2 Integrins in Neutrophils, 2(7) (2018), pp. 731-744.

[80] M. Phillipson, et al., Intraluminal crawling of neutrophils to emigration sites: a molecularly distinct process from adhesion in the recruitment cascade, J. Exp. Med. 203 (12) (2006) 2569-2575.

[81] R. Sumagin, H. Prizant, E. Lomakina, R.E. Waugh, I.H. Sarelius, LFA-1 and Mac-1 define characteristically different intralumenal crawling and emigration patterns for monocytes and neutrophils in situ, J. Immunol. (Baltimore, Md. : 1950) 185 (11) (2010) 7057-7066.

[82] W.A. Muller, How endothelial cells regulate transmigration of leukocytes in the inflammatory response, Am. J. Pathol. 184 (4) (2014) 886-896.

[83] S.K. Shaw, et al., Coordinated redistribution of leukocyte LFA-1 and endothelial cell ICAM-1 accompany neutrophil transmigration, J. Exp. Med. 200 (12) (2004) $1571-1580$.

[84] O. Barreiro, et al., Dynamic interaction of VCAM-1 and ICAM-1 with moesin and ezrin in a novel endothelial docking structure for adherent leukocytes, J. Cell Biol. 157 (7) (2002) 1233-1245.

[85] S. Wang, et al., Venular basement membranes contain specific matrix protein low expression regions that act as exit points for emigrating neutrophils, J. Exp. Med. 203 (6) (2006) 1519-1532.

[86] M.B. Voisin, A. Woodfin, S. Nourshargh, Monocytes and neutrophils exhibit both distinct and common mechanisms in penetrating the vascular basement membrane in vivo, Arterioscler. Thromb. Vasc. Biol. 29 (8) (2009) 1193-1199.

[87] R. Hallmann, et al., The regulation of immune cell trafficking by the extracellular matrix, Curr. Opin. Cell Biol. 36 (2015) 54-61.

[88] K. Ley, C. Laudanna, M.I. Cybulsky, S. Nourshargh, Getting to the site of in flammation: the leukocyte adhesion cascade updated, Nat. Rev. Immunol. 7 (9) (2007) 678-689.

[89] L.C. Kelley, L.L. Lohmer, E.J. Hagedorn, D.R. Sherwood, Traversing the basement membrane in vivo: a diversity of strategies, J. Cell Biol. 204 (3) (2014) 291.

[90] R.O. Hynes, The extracellular matrix: not just pretty fibrils, Science (New York, N.Y.) 326 (5957) (2009) 1216-1219.

[91] I. Stamenkovic, Extracellular matrix remodelling: the role of matrix metalloproteinases, J. Pathol. 200 (4) (2003) 448-464.

[92] G.G. Vaday, et al., Combinatorial signals by inflammatory cytokines and chemokines mediate leukocyte interactions with extracellular matrix, J. Leukoc. Biol. 69 (6) (2001) 885-892.

[93] T. Vorup-Jensen, et al., Exposure of acidic residues as a danger signal for recognition of fibrinogen and other macromolecules by integrin alphaXbeta2, Proc. Natl. Acad. Sci. U. S. A. 102 (5) (2005) 1614-1619.

[94] K. Wolf, R. Muller, S. Borgmann, E.B. Brocker, P. Friedl, Amoeboid shape change and contact guidance: T-lymphocyte crawling through fibrillar collagen is independent of matrix remodeling by MMPs and other proteases, Blood 102 (9)
(2003) 3262-3269.

[95] K. Wolf, et al., Physical limits of cell migration: control by ECM space and nuclear deformation and tuning by proteolysis and traction force, J. Cell Biol. 201 (7) (2013) 1069-1084.

[96] B.A. David, P. Kubes, Exploring the complex role of chemokines and chemoattractants in vivo on leukocyte dynamics, Immunol. Rev. 289 (1) (2019) 9-30.

[97] D.F. Legler, M. Thelen, Chemokines: chemistry, biochemistry and biological function, Chimia 70 (12) (2016) 856-859.

[98] A.E. Proudfoot, et al., Glycosaminoglycan binding and oligomerization are essential for the in vivo activity of certain chemokines, Proc. Natl. Acad. Sci. U. S. A. 100 (4) (2003) 1885-1890.

[99] B. Heit, S. Tavener, E. Raharjo, P. Kubes, An intracellular signaling hierarchy determines direction of migration in opposing chemotactic gradients, J. Cell Biol. 159 (1) (2002) 91-102.

[100] H. Lortat-Jacob, A. Grosdidier, A. Imberty, Structural diversity of heparan sulfate binding domains in chemokines, Proc. Natl. Acad. Sci. 99 (3) (2002) 1229.

[101] M. Binnewies, et al., Understanding the tumor immune microenvironment (TIME) for effective therapy, Nat. Med. 24 (5) (2018) 541-550.

[102] G. Bindea, et al., Spatiotemporal dynamics of intratumoral immune cells reveal the immune landscape in human cancer, Immunity 39 (4) (2013) 782-795.

[103] S. Hendry, et al., Assessing tumor-infiltrating lymphocytes in solid tumors: a practical review for pathologists and proposal for a standardized method from the International Immunooncology Biomarkers Working Group: part 1: assessing the host immune response, TILs in invasive breast carcinoma and ductal carcinoma in situ, metastatic tumor deposits and areas for further research, Adv. Anat. Pathol. 24 (5) (2017) 235-251.

[104] S. Spranger, Mechanisms of tumor escape in the context of the T-cell-inflamed and the non-T-cell-inflamed tumor microenvironment, Int. Immunol. 28 (8) (2016) 383-391.

[105] J.C. Roth, D.T. Curiel, L. Pereboeva, Cell vehicle targeting strategies, Gene Ther. 15 (2008) 716.

[106] O.S. Blomberg, L. Spagnuolo, K.E. de Visser, Immune regulation of metastasis: mechanistic insights and therapeutic opportunities, Dis. Model Mech. 11 (10) (2018) (dmm036236).

[107] W.A. Lim, C.H. June, The principles of engineering immune cells to treat cancer, Cell 168 (4) (2017) 724-740.

[108] M.A. Fischbach, J.A. Bluestone, W.A. Lim, Cell-based therapeutics: the next pillar of medicine, Sci. Transl. Med. 5 (179) (2013) (179ps177).

[109] W.A. Lim, Designing customized cell signalling circuits, Nat. Rev. Mol. Cell. Biol. 11 (6) (2010) 393-403.

[110] K. Harrington, et al., Cells as vehicles for cancer gene therapy: the missing link between targeted vectors and systemic delivery? Hum. Gene Ther. 13 (11) (2002) $1263-1280$

[111] M. Hamidi, A. Zarrin, M. Foroozesh, S. Mohammadi-Samani, Applications of carrier erythrocytes in delivery of biopharmaceuticals, J. Control. Release 118 (2) (2007) 145-160.

[112] K. Kinosita, T.Y. Tsong, Survival of sucrose-loaded erythrocytes in the circulation, Nature 272 (5650) (1978) 258-260.

[113] C.H. Villa, et al., Delivery of drugs bound to erythrocytes: new avenues for an old intravascular carrier, Ther. Deliv. 6 (7) (2015) 795-826.

[114] F. Pierigè, N. Bigini, L. Rossi, M. Magnani, Reengineering red blood cells for cellular therapeutics and diagnostics, Wiley Interdiscip. Rev. 9 (5) (2017) e1454.

[115] E. Lee, et al., The RNA in reticulocytes is not just debris: it is necessary for the final stages of erythrocyte formation, Blood Cells Mol. Dis. 53 (1-2) (2014) 1-10.

[116] F.J. Alvarez, et al., Cross-linking treatment of loaded erythrocytes increases delivery of encapsulated substance to macrophages, Biotechnol. Appl. Biochem. 27 (2) (1998) 139-143.

[117] F.J. Alvarez, J.A. Jordan, A. Herraez, J.C. Diez, M.C. Tejedor, Hypotonically loaded rat erythrocytes deliver encapsulated substances into peritoneal macrophages, J. Biochem. 123 (2) (1998) 233-239.

[118] S.G. Moreno, Depleting macrophages in vivo with clodronate-liposomes, Methods Mol. Biol. (Clifton, N.J.) 1784 (2018) 259-262.

[119] P. Burger, P. Hilarius-Stokman, D. de Korte, T.K. van den Berg, R. van Bruggen, CD47 functions as a molecular switch for erythrocyte phagocytosis, Blood 119 (23) (2012) 5512-5521.

[120] E. Bashar, K. Gregory-Evans, Magnetic Nanoparticles in Cell-based Therapies, (2016), pp. 161-184.

[121] R.J. Price, D.M. Skyba, S. Kaul, T.C. Skalak, Delivery of colloidal particles and red blood cells to tissue through microvessel ruptures created by targeted microbubble destruction with ultrasound, Circulation 98 (13) (1998) 1264-1267.

[122] V.N. Smirnov, et al., Carrier-directed targeting of liposomes and erythrocytes to denuded areas of vessel wall, Proc. Natl. Acad. Sci. U. S. A. 83 (17) (1986) $6603-6607$.

[123] H. Vink, B.R. Duling, Identification of distinct luminal domains for macromolecules, erythrocytes, and leukocytes within mammalian capillaries, Circ. Res. 79 (3) (1996) 581-589.

[124] A. Lucas, D. Lam, P. Cabrales, Doxorubicin-loaded red blood cells reduced cardiac toxicity and preserved anticancer activity, Drug Deliv. 26 (1) (2019) 433-442.

[125] N.M. La-Beck, A.A. Gabizon, Nanoparticle interactions with the immune system: clinical implications for liposome-based cancer chemotherapy, Front. Immunol. 8 (2017) 416

[126] Y. Lu, Q. Hu, C. Jiang, Z. Gu, Platelet for drug delivery, Curr. Opin. Biotechnol. 58 (2019) 81-91.

[127] J.E. Italiano Jr.et al., Angiogenesis is regulated by a novel mechanism: pro- and antiangiogenic proteins are organized into separate platelet alpha granules and differentially released, Blood 111 (3) (2008) 1227-1233. 
[128] N. Ortiz-Otero, Z. Mohamed, M.R. King, Platelet-based drug delivery for cancer applications, Adv. Exp. Med. Biol. 1092 (2018) 235-251.

[129] T. Burnouf, H.A. Goubran, M.L. Chou, D. Devos, M. Radosevic, Platelet microparticles: detection and assessment of their paradoxical functional roles in disease and regenerative medicine, Blood Rev. 28 (4) (2014) 155-166.

[130] D. Buergy, F. Wenz, C. Groden, M.A. Brockmann, Tumor-platelet interaction in solid tumors, Int. J. Cancer 130 (12) (2012) 2747-2760.

[131] B. Ho-Tin-Noe, T. Goerge, S.M. Cifuni, D. Duerschmied, D.D. Wagner, Platelet granule secretion continuously prevents intratumor hemorrhage, Cancer Res. 68 (16) (2008) 6851-6858.

[132] P. Xu, et al., Doxorubicin-loaded platelets as a smart drug delivery system: an improved therapy for lymphoma, Sci. Rep. 7 (2017) 42632

[133] P. Xu, et al., Doxorubicin-loaded platelets conjugated with anti-CD22 mAbs: a novel targeted delivery system for lymphoma treatment with cardiopulmonary avoidance, Oncotarget 8 (35) (2017) 58322-58337.

[134] Y. Zhang, G. Liu, J. Wei, G. Nie, Platelet membrane-based and tumor-associated platelettargeted drug delivery systems for cancer therapy, Front. Med. 12 (6) (2018) 667-677.

[135] C. Wang, et al., In situ activation of platelets with checkpoint inhibitors for postsurgical cancer immunotherapy, Nat. Biomed. Eng. 1 (2017) 0011.

[136] X. Han, et al., Platelets as platforms for inhibition of tumor recurrence post-phy sical therapy by delivery of anti-PD-L1 checkpoint antibody, J. Control. Release 304 (2019) 233-241.

[137] J.L. Sieow, S.Y. Gun, S.C. Wong, The sweet surrender: how myeloid cell metabolic plasticity shapes the tumor microenvironment, Front. Cell Dev. Biol. 6 (168) (2018).

[138] R.M. Awad, Y. De Vlaeminck, J. Maebe, C. Goyvaerts, K. Breckpot, Turn back the TIMe: targeting tumor infiltrating myeloid cells to revert cancer progression, Front. Immunol. 9 (2018) 1977.

[139] D. Hanahan, R.A. Weinberg, Hallmarks of cancer: the next generation, Cell 144 (5) (2011) 646-674.

[140] M. Karin, F.R. Greten, NF-kappaB: linking inflammation and immunity to cancer development and progression, Nat. Rev. Immunol. 5 (10) (2005) 749-759.

[141] F. Veglia, M. Perego, Myeloid-derived Suppressor Cells Coming of Age, 19(2) (2018), pp. 108-119.

[142] M. Kiss, S. Van Gassen, K. Movahedi, Y. Saeys, D. Laoui, Myeloid cell heterogeneity in cancer: not a single cell alike, Cell. Immunol. 330 (2018) 188-201.

[143] H. Peinado, S. Lavotshkin, D. Lyden, The secreted factors responsible for premetastatic niche formation: old sayings and new thoughts, Semin. Cancer Biol. 21 (2) (2011) 139-146.

[144] J. Jablonska, S. Lang, R.V. Sionov, Z. Granot, The regulation of pre-metastatic niche formation by neutrophils, Oncotarget 8 (67) (2017) 112132-112144.

[145] T. Loos, A. Mortier, P. Proost, Chapter 1. Isolation, identification, and production of posttranslationally modified chemokines, Methods Enzymol. 461 (2009) 3-29.

[146] A. Mortier, J. Van Damme, P. Proost, Regulation of chemokine activity by posttranslational modification, Pharmacol. Ther. 120 (2) (2008) 197-217.

[147] B. Molon, et al., Chemokine nitration prevents intratumoral infiltration of antigenspecific T cells, J. Exp. Med. 208 (10) (2011) 1949-1962.

[148] D. Chu, X. Dong, X. Shi, C. Zhang, Z. Wang, Neutrophil-based drug delivery systems, Adv. Mater. 30 (22) (2018) e1706245.

[149] C. Summers, et al., Neutrophil kinetics in health and disease, Trends Immunol. 31 (8) (2010) 318-324.

[150] J.M. Petty, C.C. Lenox, D.J. Weiss, M.E. Poynter, B.T. Suratt, Crosstalk between CXCR4/stromal derived factor-1 and VLA-4/VCAM-1 pathways regulates neutrophil retention in the bone marrow, J. Immunol. (Baltimore, Md. : 1950) 182 (1) (2009) 604-612.

[151] A.E. Ekpenyong, N. Toepfner, E.R. Chilvers, J. Guck, Mechanotransduction in neutrophil activation and deactivation, Biochim. Biophys. Acta 11 (Part B) (2015) 3105-3116.

[152] A.M. Mauer, J.W. Athens, H. Ashenbrucker, G.E. Cartwright, M.M. Wintrobe, Leukokinetic studies. II. A method for labeling granulocytes in vitro with radioactive diisopropylfluorophosphate (DFP), J. Clin. Invest. 39 (9) (1960) 1481-1486.

[153] J.W. Athens, et al., Leukokinetic studies. III. The distribution of granulocytes in the blood of normal subjects, J. Clin. Investig. 40 (1961) 159-164.

[154] J.W. Athens, et al., Leukokinetic studies. IV. The total blood, circulating and marginal granulocyte pools and the granulocyte turnover rate in normal subjects, J. Clin. Invest. 40 (1961) 989-995.

[155] W. MacNee, C. Selby, New perspectives on basic mechanisms in lung disease. 2. Neutrophil traffic in the lungs: role of haemodynamics, cell adhesion, and deformability, Thorax 48 (1) (1993) 79-88.

[156] C. Summers, et al., Neutrophil kinetics in health and disease, Trends Immunol. 31 (8) (2010) 318-324.

[157] W.M. Kuebler, A.E. Goetz, The marginated pool, Eur. Surg. Res. 34 (1-2) (2002) 92-100.

[158] B.A. Martin, J.L. Wright, H. Thommasen, J.C. Hogg, Effect of pulmonary blood flow on the exchange between the circulating and marginating pool of polymorphonuclear leukocytes in dog lungs, J. Clin. Invest. 69 (6) (1982) 1277-1285.

[159] C.M. Doerschuk, N. Beyers, H.O. Coxson, B. Wiggs, J.C. Hogg, Comparison of neutrophil and capillary diameters and their relation to neutrophil sequestration in the lung, J. Appl. Physiol. (Bethesda, Md. : 1985) 74 (6) (1993) 3040-3045.

[160] B.T. Suratt, et al., Neutrophil maturation and activation determine anatomic site of clearance from circulation, Am. J. Physiol. 281 (4) (2001) L913-L.921.

[161] J. Shi, G.E. Gilbert, Y. Kokubo, T. Ohashi, Role of the liver in regulating numbers of circulating neutrophils, Blood 98 (4) (2001) 1226-1230.

[162] J. Grommes, O. Soehnlein, Contribution of neutrophils to acute lung injury, Mol.
Med. 17 (3-4) (2011) 293-307.

[163] J.M. Petty, et al., Pulmonary stromal-derived factor-1 expression and effect on neutrophil recruitment during acute lung injury, J. Immunol. (Baltimore, Md. : 1950) 178 (12) (2007) 8148-8157.

[164] I. Miralda, S.M. Uriarte, K.R. McLeish, Multiple phenotypic changes define neutrophil priming, Front. Cell. Infect. Microbiol. 7 (2017) 217.

[165] R.S. Frank, Time-dependent alterations in the deformability of human neutrophils in response to chemotactic activation, Blood 76 (12) (1990) 2606

[166] C. Summers, et al., Pulmonary retention of primed neutrophils: a novel protective host response, which is impaired in the acute respiratory distress syndrome, Thorax 69 (7) (2014) 623-629.

[167] S. de Oliveira, et al., Cxcl8 (IL-8) mediates neutrophil recruitment and behavior in the zebrafish inflammatory response, J. Immunol. (Baltimore, Md. : 1950) 190 (8) (2013) 4349-4359.

[168] A.M. Condliffe, E.R. Chilvers, C. Haslett, I. Dransfield, Priming differentially reg ulates neutrophil adhesion molecule expression/function, Immunology 89 (1) (1996) 105-111.

[169] K.L. Vogt, C. Summers, E.R. Chilvers, Priming and De-priming of Neutrophil Responses In Vitro and In Vivo, 48 (2) (2018), p. e12967.

[170] J.M. Paulsson, S.H. Jacobson, J. Lundahl, Neutrophil activation during transmigration in vivo and in vitro A translational study using the skin chamber model, J. Immunol. Methods 361 (1-2) (2010) 82-88.

[171] T.N. Mayadas, X. Cullere, C.A. Lowell, The multifaceted functions of neutrophils, Annu. Rev. Pathol. 9 (2014) 181-218.

[172] C. Eyileten, et al., Immune cells in cancer therapy and drug delivery, Mediat Inflamm. 2016 (2016) 5230219.

[173] E.B. Eruslanov, S. Singhal, S.M. Albelda, Mouse versus human neutrophils in cancer: a major knowledge gap, Trends Cancer 3 (2) (2017) 149-160.

[174] G.B. Segel, M.W. Halterman, M.A. Lichtman, The paradox of the neutrophil's role in tissue injury, J. Leukoc. Biol. 89 (3) (2011) 359-372.

[175] S.O. Wendel, et al., Cell based drug delivery: micrococcus luteus loaded neutrophils as chlorhexidine delivery vehicles in a mouse model of liver abscesses in cattle, PLoS One 10 (5) (2015) e0128144.

[176] D. Chu, X. Dong, X. Shi, C. Zhang, Z. Wang, Neutrophil. Based Drug Deliv. Syst. 30 (22) (2018) e1706245.

[177] D. Chu, X. Dong, Q. Zhao, J. Gu, Z. Wang, Photosensitization priming of tumo microenvironments improves delivery of nanotherapeutics via neutrophil infiltration, Adv. Mater. 29 (27) (2017) 1701021.

[178] J. Xue, et al., Neutrophil-mediated anticancer drug delivery for suppression of postoperative malignant glioma recurrence, Nat. Nanotechnol. 12 (7) (2017) 692-700.

[179] G.A. Ramirez, et al., Eosinophils from physiology to disease: a comprehensive review, Biomed. Res. Int. 2018 (2018) 9095275.

[180] K.D. Stone, C. Prussin, D.D. Metcalfe, IgE, mast cells, basophils, and eosinophils, J. Allergy Clin. Immunol. 125 (2 Suppl 2) (2010) S73-S80.

[181] J.J. Lee, E.A. Jacobsen, M.P. McGarry, R.P. Schleimer, N.A. Lee, Eosinophils in health and disease: the LIAR hypothesis, Clin. Exp. Allergy 40 (4) (2010) 563-575.

[182] S.A. Cormier, et al., Pivotal advance: eosinophil infiltration of solid tumors is an early and persistent inflammatory host response, J. Leukoc. Biol. 79 (6) (2006) $1131-1139$.

[183] T. Wen, J.A. Besse, M.K. Mingler, P.C. Fulkerson, M.E. Rothenberg, Eosinophil adoptive transfer system to directly evaluate pulmonary eosinophil trafficking in vivo, Proc. Natl. Acad. Sci. U. S. A. 110 (15) (2013) 6067-6072.

[184] A.K. Johansson, S. Sergejeva, M. Sjostrand, J.J. Lee, J. Lotvall, Allergen-induced traffic of bone marrow eosinophils, neutrophils and lymphocytes to airways, Eur. J. Immunol. 34 (11) (2004) 3135-3145.

[185] N. Charles, J.M. Chemouny, E. Daugas, Basophil involvement in lupus nephritis: a basis for innovation in daily care, Nephrol. Dial. Transplant. 34 (5) (2018) 750-756.

[186] B. Min, M.A. Brown, G. Legros, Understanding the roles of basophils: breaking dawn, Immunology 135 (3) (2012) 192-197.

[187] H. Karasuyama, K. Mukai, K. Obata, Y. Tsujimura, T. Wada, Nonredundant roles of basophils in immunity, Annu. Rev. Immunol. 29 (2011) 45-69.

[188] M.C. Siracusa, et al., TSLP promotes interleukin-3-independent basophil haematopoiesis and type 2 inflammation, Nature 477 (7363) (2011) 229-233.

[189] M. Iikura, et al., Chemokine receptors in human basophils: inducible expression of functional CXCR4, J. Leukoc. Biol. 70 (1) (2001) 113-120.

[190] S. Yona, et al., Fate mapping reveals origins and dynamics of monocytes and tissue macrophages under homeostasis, Immunity 38 (1) (2013) 79-91.

[191] D. Hashimoto, et al., Tissue-resident macrophages self-maintain locally throughout adult life with minimal contribution from circulating monocytes, Immunity 38 (4) (2013) 792-804.

[192] K. Liu, et al., In vivo analysis of dendritic cell development and homeostasis, Science (New York, N.Y.) 324 (5925) (2009) 392.

[193] C. Auffray, et al., Monitoring of blood vessels and tissues by a population of monocytes with patrolling behavior, Science (New York, N.Y.) 317 (5838) (2007) $666-670$.

[194] F. Ginhoux, S. Jung, Monocytes and macrophages: developmental pathways and tissue homeostasis, Nat. Rev. Immunol. 14 (2014) 392.

[195] N.V. Serbina, E.G. Pamer, Monocyte emigration from bone marrow during bac terial infection requires signals mediated by chemokine receptor CCR2, Nat. Immunol. 7 (3) (2006) 311-317.

[196] C.S. Robbins, et al., Extramedullary hematopoiesis generates Ly-6C(high) monocytes that infiltrate atherosclerotic lesions, Circulation 125 (2) (2012) 364-374.

[197] F.K. Swirski, et al., Identification of splenic reservoir monocytes and their deployment to inflammatory sites, Science (New York, N.Y.) 325 (5940) (2009) 
612-616.

[198] K.P. O’Dea, et al., Mobilization and margination of bone marrow Gr-1high monocytes during subclinical endotoxemia predisposes the lungs toward acute injury, J. Immunol. (Baltimore, Md. : 1950) 182 (2) (2009) 1155-1166.

[199] K.E. O'Connell, et al., Practical murine hematopathology: a comparative review and implications for research, Comp. Med. 65 (2) (2015) 96-113.

[200] P.Y. Lee, J.X. Wang, E. Parisini, C.C. Dascher, P.A. Nigrovic, Ly6 family proteins in neutrophil biology, J. Leukoc. Biol. 94 (4) (2013) 585-594.

[201] F. Geissmann, S. Jung, D.R. Littman, Blood monocytes consist of two principal subsets with distinct migratory properties, Immunity 19 (1) (2003) 71-82.

[202] A. Mildner, et al., Genomic characterization of murine monocytes reveals C/ EBPbeta transcription factor dependence of Ly6C(-) cells, Immunity 46 (5) (2017) 849-862 (e847).

[203] C. Varol, et al., Monocytes give rise to mucosal, but not splenic, conventional dendritic cells, J. Exp. Med. 204 (1) (2007) 171-180.

[204] A. Mildner, et al., CCR2 + Ly-6Chi monocytes are crucial for the effector phase of autoimmunity in the central nervous system, Brain J. Neurol. 132 (Pt 9) (2009) 2487-2500.

[205] P.J. Murray, Macrophage Polarization, Annu. Rev. Physiol. 79 (1) (2017) 541-566.

[206] L.C. Davies, S.J. Jenkins, J.E. Allen, P.R. Taylor, Tissue-resident macrophages, Nat. Immunol. 14 (10) (2013) 986-995.

[207] S.J. Jenkins, et al., Local macrophage proliferation, rather than recruitment from the blood, is a signature of TH2 inflammation, Science (New York, N.Y.) 332 (6035) (2011) 1284-1288.

[208] S.J. Jenkins, et al., IL-4 directly signals tissue-resident macrophages to proliferate beyond homeostatic levels controlled by CSF-1, J. Exp. Med. 210 (11) (2013) 2477.

[209] C. Varol, et al., Intestinal lamina propria dendritic cell subsets have different origin and functions, Immunity 31 (3) (2009) 502-512.

[210] C.S. Robbins, et al., Local proliferation dominates lesional macrophage accumulation in atherosclerosis, Nat. Med. 19 (2013) 1166.

[211] T. Jia, et al., Additive roles for MCP-1 and MCP-3 in CCR2-mediated recruitment of inflammatory monocytes during $<\mathrm{em}>$ listeria monocytogenes $</$ em $>$ infection, J. Immunol. 180 (10) (2008) 6846.

[212] T. Gerhardt, K. Ley, Monocyte trafficking across the vessel wall, Cardiovasc. Res. 107 (3) (2015) 321-330.

[213] C.L. Maslin, K. Kedzierska, N.L. Webster, W.A. Muller, S.M. Crowe, Transendothelial migration of monocytes: the underlying molecular mechanisms and consequences of HIV-1 infection, Curr. HIV Res. 3 (4) (2005) 303-317.

[214] C. Shi, E.G. Pamer, Monocyte recruitment during infection and inflammation, Nat. Rev. Immunol. 11 (11) (2011) 762-774.

[215] R.H. Wiltrout, et al., Distribution of peritoneal macrophage populations after intravenous injection in mice: differential effects of eliciting and activating agents, J. Reticuloendothel. Soc. 34 (3) (1983) 253-269.

[216] R. Audran, B. Collet, A. Moisan, L. Toujas, Fate of mouse macrophages radiolabelled with PKH-95 and injected intravenously, Nucl. Med. Biol. 22 (6) (1995) 817-821.

[217] Y. Sawanobori, et al., Chemokine-mediated rapid turnover of myeloid-derived suppressor cells in tumor-bearing mice, Blood 111 (12) (2008) 5457-5466.

[218] T. Lesimple, A. Moisan, L. Toujas, Autologous human macrophages and anti-tumour cell therapy, Res. Immunol. 149 (7-8) (1998) 663-671.

[219] R. Andreesen, B. Hennemann, S.W. Krause, Adoptive immunotherapy of cancer using monocyte-derived macrophages: rationale, current status, and perspectives, J. Leukoc. Biol. 64 (4) (1998) 419-426.

[220] B.A. Teicher, Hypoxia and drug resistance, Cancer Metastasis Rev. 13 (2) (1994) $139-168$.

[221] D. Lindsay, C.M. Garvey, S.M. Mumenthaler, J. Foo, Leveraging hypoxia-activated prodrugs to prevent drug resistance in solid tumors, PLoS Comput. Biol. 12 (8) (2016) e1005077.

[222] P.J. Bugelski, R. Kirsh, C. Buscarino, S.P. Corwin, G. Poste, Recruitment of exogenous macrophages into metastases at different stages of tumor growth, Cancer Immunol. Immunother. 24 (2) (1987) 93-98.

[223] R. Parsa, et al., Adoptive transfer of immunomodulatory M2 macrophages prevents type 1 diabetes in NOD mice, Diabetes 61 (11) (2012) 2881-2892.

[224] J.H. Seo, et al., Trafficking macrophage migration using reporter gene imaging with human sodium iodide symporter in animal models of inflammation, J. Nuclear Med. 51 (10) (2010) 1637-1643.

[225] L. Griffiths, et al., The macrophage - a novel system to deliver gene therapy to pathological hypoxia, Gene Ther. 7 (3) (2000) 255-262.

[226] L. Carta, et al., Engineering of macrophages to produce IFN-gamma in response to hypoxia, J. Immunol. (Baltimore, Md. : 1950) 166 (9) (2001) 5374-5380.

[227] R. Andreesen, B. Hennemann, S.W. Krause, Adoptive immunotherapy of cancer using monocyte-derived macrophages: rationale, current status, and perspectives, J. Leukoc. Biol. 64 (4) (1998) 419-426.

[228] M. Muthana, et al., Use of macrophages to target therapeutic adenovirus to human prostate tumors, Cancer Res. 71 (5) (2011) 1805-1815.

[229] D.G. Roy, J.C. Bell, Cell carriers for oncolytic viruses: current challenges and future directions, Oncolyt. Virother. 2 (2013) 47-56.

[230] M.A. Boemo, H.M. Byrne, Mathematical modelling of a hypoxia-regulated oncolytic virus delivered by tumour-associated macrophages, J. Theor. Biol. 461 (2019) 102-116.

[231] P.S. Jiang, et al., Irradiation enhances the ability of monocytes as nanoparticle carrier for cancer therapy, PLoS One 10 (9) (2015) e0139043.

[232] T.D. McKee, et al., Degradation of fibrillar collagen in a human melanoma xenograft improves the efficacy of an oncolytic herpes simplex virus vector, Cancer Res.
66 (5) (2006) 2509

[233] G. Pawelec, C.P. Verschoor, S. Ostrand-Rosenberg, Myeloid-derived suppressor cells: not only in tumor immunity, Front. Immunol. 10 (2019) 1099.

[234] D.I. Gabrilovich, et al., The terminology issue for myeloid-derived suppressor cells, Cancer Res. 67 (1) (2007) 425-426.

[235] V. Bronte, et al., Recommendations for myeloid-derived suppressor cell nomenclature and characterization standards, Nat. Commun. 7 (2016) 12150.

[236] A.M. Bruger, et al., How to measure the immunosuppressive activity of, MDSC 68 (4) (2019) 631-644.

[237] L. Cassetta, et al., Deciphering myeloid-derived suppressor cells: isolation and markers in humans, mice and non-human primates, Cancer Immunol. Immunother. 68 (4) (2019) 687-697.

[238] V. Bronte, et al., Recommendations for myeloid-derived suppressor cell nomenclature and characterization standards, Nat. Commun. 7 (2016) 12150.

[239] D.I. Gabrilovich, S. Ostrand-Rosenberg, V. Bronte, Coordinated regulation of myeloid cells by tumours, Nat. Rev. Immunol. 12 (4) (2012) 253-268.

[240] S. Brandau, et al., Myeloid-derived suppressor cells in the peripheral blood of cancer patients contain a subset of immature neutrophils with impaired migratory properties, J. Leukoc. Biol. 89 (2) (2011) 311-317.

[241] I.H. Younos, A.J. Dafferner, D. Gulen, H.C. Britton, J.E. Talmadge, Tumor regulation of myeloid-derived suppressor cell proliferation and trafficking, Int Immunopharmacol. 13 (3) (2012) 245-256.

[242] V. Cortez-Retamozo, et al., Origins of tumor-associated macrophages and neutrophils, Proc. Natl. Acad. Sci. U. S. A. 109 (7) (2012) 2491-2496.

[243] L. Levy, et al., Splenectomy inhibits non-small cell lung cancer growth by modulating anti-tumor adaptive and innate immune response, Oncoimmunology 4 (4) (2015) e998469.

[244] B. Li, et al., CCL9/CCR1 induces myeloidderived suppressor cell recruitment to the spleen in a murine H22 orthotopic hepatoma model, Oncol. Rep. 41 (1) (2019) 608-618.

[245] S.L. Highfill, et al., Disruption of CXCR2-mediated MDSC tumor trafficking enhances anti-PD1 efficacy, Sci. Transl. Med. 6 (237) (2014) (237ra267).

[246] L. Sun, et al., Inhibiting myeloid-derived suppressor cell trafficking enhances T cell immunotherapy, JCI Insight 4 (7) (2019) e126853.

[247] X. Lu, et al., Effective combinatorial immunotherapy for castration-resistant prostate cancer, Nature 543 (7647) (2017) 728-732.

[248] I. Marigo, et al., Tumor-induced tolerance and immune suppression depend on the C/EBPbeta transcription factor, Immunity 32 (6) (2010) 790-802.

[249] J. Sceneay, et al., Tracking the Fate of Adoptively Transferred Myeloid-derived Suppressor Cells in the Primary Breast Tumor Microenvironment, 13 (4) (2018) e0196040.

[250] F. Lassailly, E. Griessinger, D. Bonnet, "Microenvironmental contaminations" induced by fluorescent lipophilic dyes used for noninvasive in vitro and in vivo cell tracking, Blood 115 (26) (2010) 5347-5354.

[251] S. Eisenstein, et al., Myeloid-derived suppressor cells as a vehicle for tumor-specific oncolytic viral therapy, Cancer Res. 73 (16) (2013) 5003-5015.

[252] D. Chandra, A. Jahangir, W. Quispe-Tintaya, M.H. Einstein, C. Gravekamp, Myeloid-derived suppressor cells have a central role in attenuated Listeria monocytogenes-based immunotherapy against metastatic breast cancer in young and old mice, Br. J. Cancer 108 (11) (2013) 2281-2290.

[253] S.H. Kim, F. Castro, Y. Paterson, C. Gravekamp, High efficacy of a Listeria-based vaccine against metastatic breast cancer reveals a dual mode of action, Cancer Res. 69 (14) (2009) 5860-5866.

[254] K. Newick, S. O'Brien, E. Moon, S.M. Albelda, CAR T cell therapy for solid tumors, Annu. Rev, Med, 68 (2017) 139-152.

[255] Z. Wang, W. Chen, X. Zhang, Z. Cai, W. Huang, A long way to the battlefront: CAR T cell therapy against solid cancers, J. Cancer 10 (14) (2019) 3112-3123.

[256] J.D. Peske, A.B. Woods, V.H. Engelhard, Control of CD8 T-cell infiltration into tumors by vasculature and microenvironment, Adv. Cancer Res. 128 (2015) 263-307.

[257] M. Idorn, P. Thor Straten, Chemokine receptors and exercise to tackle the inadequacy of T cell homing to the tumor site, Cells 7 (8) (2018).

[258] K. Suzuki, A. Nakai, Control of lymphocyte trafficking and adaptive immunity by adrenergic nerves, Clin. Exp. Neuroimmunol. 8 (1) (2017) 15-22.

[259] M.W. Rohaan, S. Wilgenhof, J.B.A.G. Haanen, Adoptive cellular therapies: the current landscape, Virchows Arch. 474 (4) (2019) 449-461.

[260] B.L. Levine, J. Miskin, K. Wonnacott, C. Keir, Global manufacturing of CAR T cell therapy, Mol. Ther. Methods Clin. Dev. 4 (2017) 92-101.

[261] J.P. Girard, C. Moussion, R. Forster, HEVs, lymphatics and homeostatic immune cell trafficking in lymph nodes, Nat. Rev. Immunol. 12 (11) (2012) 762-773.

[262] N. Garbi, B. Arnold, S. Gordon, G.J. Hammerling, R. Ganss, CpG motifs as proin flammatory factors render autochthonous tumors permissive for infiltration and destruction, J. Immunol. (Baltimore, Md. : 1950) 172 (10) (2004) 5861-5869.

[263] R.A. Clark, et al., Human squamous cell carcinomas evade the immune response by down-regulation of vascular E-selectin and recruitment of regulatory T cells, J. Exp. Med. 205 (10) (2008) 2221-2234.

[264] M.H. Kershaw, et al., Redirecting migration of T cells to chemokine secreted from tumors by genetic modification with CXCR2, Hum. Gene Ther. 13 (16) (2002) 1971-1980.

[265] I. Siddiqui, M. Erreni, M. van Brakel, R. Debets, P. Allavena, Enhanced recruitmen of genetically modified CX3CR1-positive human T cells into Fractalkine/CX3CL1 expressing tumors: importance of the chemokine gradient, J. Immunother. Cancer 4 (2016) 21.

[266] A. Di Stasi, et al., T lymphocytes coexpressing CCR4 and a chimeric antigen receptor targeting CD30 have improved homing and antitumor activity in a Hodgkin tumor model, Blood 113 (25) (2009) 6392-6402. 
[267] J.A. Craddock, et al., Enhanced tumor trafficking of GD2 chimeric antigen receptor $\mathrm{T}$ cells by expression of the chemokine receptor CCR2b, J. Immunother. (Hagerstown, Md. : 1997) 33 (8) (2010) 780-788.

[268] E.K. Moon, et al., Expression of a functional CCR2 receptor enhances tumor localization and tumor eradication by retargeted human $\mathrm{T}$ cells expressing a mesothelin-specific chimeric antibody receptor, Clin. Cancer Res. 17 (14) (2011) 4719-4730.

[269] M. Chmielewski, A.A. Hombach, H. Abken, Of CARs and TRUCKs: chimeric antigen receptor (CAR) T cells engineered with an inducible cytokine to modulate the tumor stroma, Immunol. Rev. 257 (1) (2014) 83-90.

[270] M. Chmielewski, C. Kopecky, A.A. Hombach, H. Abken, IL-12 release by en gineered T cells expressing chimeric antigen receptors can effectively Muster an antigen-independent macrophage response on tumor cells that have shut down tumor antigen expression, Cancer Res. 71 (17) (2011) 5697-5706.

[271] E.D. Tait Wojno, C.A. Hunter, J.S. Stumhofer, The immunobiology of the Interleukin-12 family: room for discovery, Immunity 50 (4) (2019) 851-870.

[272] S.P. Kerkar, et al., Tumor-specific CD8 $<$ sup $>+</$ sup $>$ T cells expressing interleukin-12 eradicate established cancers in lymphodepleted hosts, Cancer Res. 70 (17) (2010) 6725.

[273] L. Zhang, et al., Improving adoptive T cell therapy by targeting and controlling IL12 expression to the tumor environment, Mol. Ther. 19 (4) (2011) 751-759.

[274] R.B. Jones, et al., Antigen recognition-triggered drug delivery mediated by nanocapsule-functionalized cytotoxic T-cells, Biomaterials 117 (2017) 44-53.

[275] D. Chinnasamy, et al., Local delivery of interleukin-12 using T cells targeting VEGF receptor-2 eradicates multiple vascularized tumors in mice, Clin. Cancer Res. 18 (6) (2012) 1672-1683.

[276] S. Patel, et al., Beyond CAR T cells: other cell-based immunotherapeutic strategies against cancer, Front. Oncol. 9 (2019) 196.

[277] M. Martinez, E.K. Moon, CAR T cells for solid tumors: new strategies for finding, infiltrating, and surviving in the tumor microenvironment, Front. Immunol. 10 (2019) 128.

[278] M. Ruella, S.S. Kenderian, Next-generation chimeric antigen receptor T-cell therapy: going off the shelf, BioDrugs 31 (6) (2017) 473-481.

[279] P. Bonaventura, et al., Cold tumors: a therapeutic challenge for immunotherapy, Front. Immunol. 10 (2019) 168.

[280] F. Fang, W. Xiao, Z. Tian, NK cell-based immunotherapy for cancer, Semin. Immunol. 31 (2017) 37-54.

[281] K. Rezvani, Adoptive cell therapy using engineered natural killer cells, Bone Marrow Transplant. 54 (2) (2019) 785-788.

[282] N.S. Sta Maria, S.R. Barnes, R.E. Jacobs, In vivo monitoring of natural killer cell trafficking during tumor immunotherapy, Magn. Reson Insights 7 (2014) 15-21.

[283] R.S. Mehta, K. Rezvani, Chimeric antigen receptor expressing natural killer cells for the immunotherapy of cancer, Front. Immunol. 9 (2018) 283.

[284] R. Meier, et al., Tracking of [18F]FDG-labeled natural killer cells to HER2/neupositive tumors, Nucl. Med. Biol. 35 (5) (2008) 579-588.

[285] B. Santomasso, C. Bachier, J. Westin, K. Rezvani, E.J. Shpall, The other side of CAR T-cell therapy: cytokine release syndrome, neurologic toxicity, and financial burden, American Society of Clinical Oncology Educational Book, 39 2019, pp. 433-444.

[286] A.M. Abel, C. Yang, M.S. Thakar, S. Malarkannan, Natural killer cells: development, maturation, and clinical utilization, Front. Immunol. 9 (2018) 1869.

[287] J.J. Campbell, et al., Unique subpopulations of CD56 + NK and NK-T peripheral blood lymphocytes identified by chemokine receptor expression repertoire, J. Immunol. (Baltimore, Md. : 1950) 166 (11) (2001) 6477-6482.

[288] M. Lima, et al., Chemokine receptor expression on normal blood CD56(+) NKcells elucidates cell partners that comigrate during the innate and adaptive immune responses and identifies a transitional NK-Cell population, J Immunol Res 2015 (2015) 839684.

[289] S. Kim, et al., In vivo developmental stages in murine natural killer cell maturation, Nat. Immunol. 3 (6) (2002) 523-528.

[290] T. Tonn, et al., Treatment of patients with advanced cancer with the natural killer cell line NK-92, Cytotherapy 15 (12) (2013) 1563-1570.

[291] K. Schonfeld, et al., Selective inhibition of tumor growth by clonal NK cells expressing an ErbB2/HER2-specific chimeric antigen receptor, Mol. Ther. 23 (2) (2015) 330-338.

[292] D.A. Knorr, et al., Clinical-scale derivation of natural killer cells from human pluripotent stem cells for cancer therapy, Stem Cells Transl. Med. 2 (4) (2013) 274-283.

[293] Y. Li, D.L. Hermanson, B.S. Moriarity, D.S. Kaufman, Human iPSC-derived natural killer cells engineered with chimeric antigen receptors enhance anti-tumor activity, Cell Stem Cell 23 (2) (2018) 181-192 (e185).

[294] E.L. Siegler, Y. Zhu, P. Wang, L. Yang, Off-the-shelf CAR-NK cells for cancer immunotherapy, Cell Stem Cell 23 (2) (2018) 160-161.

[295] R.H. Goldfarb, et al., A novel drug delivery system using IL-2 activated NK cells and Zyn-linked doxorubicin, In Vivo (Athens, Greece) 14 (1) (2000) 101-104.

[296] S. Li, et al., Nanomedicine engulfed by macrophages for targeted tumor therapy, Int. J. Nanomedicine 11 (2016) 4107-4124.

[297] T. Lang, et al., Ly6Chi monocytes delivering pH-sensitive micelle loading paclitaxel improve targeting therapy of metastatic breast cancer, Adv. Funct. Mater. 27 (26) (2017) 1701093.

[298] H. Dou, et al., Macrophage delivery of nanoformulated antiretroviral drug to the brain in a murine model of neuroAIDS, J. Immunol. (Baltimore, Md. : 1950) 183 (1) (2009) 661-669.

[299] H.I. Tong, et al., Monocyte trafficking, engraftment, and delivery of nanoparticles and an exogenous gene into the acutely inflamed brain tissue - evaluations on monocyte-based delivery system for the central nervous system, PLoS One 11 (4)
(2016) e0154022.

[300] D. Chu, X. Dong, Q. Zhao, J. Gu, Z. Wang, Photosensitization Priming of Tumor Microenvironments Improves Delivery of Nanotherapeutics via Neutrophil Infiltration, 29(27) (2017).

[301] T. Takeshima, et al., Key role for neutrophils in radiation-induced antitumor immune responses: potentiation with G-CSF, Proc. Natl. Acad. Sci. U. S. A. 113 (40) (2016) 11300-11305.

[302] D. Chu, et al., Nanoparticle targeting of neutrophils for improved cancer immunotherapy, Adv. Healthc. Mater. 5 (9) (2016) 1088-1093.

[303] F. Combes, S. Mc Cafferty, E. Meyer, N.N. Sanders, Off-target and tumor-specific accumulation of monocytes, macrophages and myeloid-derived suppressor cells after systemic injection, Neoplasia (New York, N.Y.) 20 (8) (2018) 848-856.

[304] A. Fraternale, et al., Macrophage protection by addition of glutathione (GSH)loaded erythrocytes to AZT and DDI in a murine AIDS model, Antivir. Res. 56 (3) (2002) 263-272.

[305] V.R. Muzykantov, M.D. Smirnov, A.L. Klibanov, Avidin attachment to red blood cells via a phoshpolipid derivative of biotin provides complement-resistant immunoerythrocytes, J. Immunol. Methods 158 (2) (1993) 183-190.

[306] E. Bohmova, et al., Cell-penetrating peptides: a useful tool for the delivery of various cargoes into cells, Physiol. Res. 67 (Suppl. 2) (2018) S267-s279.

[307] C.M. Hu, et al., Erythrocyte membrane-camouflaged polymeric nanoparticles as a biomimetic delivery platform, Proc. Natl. Acad. Sci. U. S. A. 108 (27) (2011) 10980-10985.

[308] A. Parodi, et al., Synthetic nanoparticles functionalized with biomimetic leukocyte membranes possess cell-like functions, Nat. Nanotechnol. 8 (1) (2013) 61-68.

[309] H. Zhang, et al., Biointerface engineering nanoplatforms for cancer-targeted drug delivery, Asian J. Pharm. Sci. (2019) (in press).

[310] A.C. Anselmo, S. Mitragotri, Cell-mediated delivery of nanoparticles: taking advantage of circulatory cells to target nanoparticles, J. Control. Release 190 (2014) 531-541.

[311] A.C. Anselmo, et al., Delivering nanoparticles to lungs while avoiding liver and spleen through adsorption on red blood cells, ACS Nano 7 (12) (2013) 11129-11137.

[312] X. Chen, et al., Interfacing carbon nanotubes with living cells, J. Am. Chem. Soc. 128 (19) (2006) 6292-6293.

[313] M.T. Stephan, J.J. Moon, S.H. Um, A. Bershteyn, D.J. Irvine, Therapeutic cell engineering with surface-conjugated synthetic nanoparticles, Nat. Med. 16 (9) (2010) 1035-1041.

[314] D. Sarkar, et al., Chemical engineering of mesenchymal stem cells to induce a cell rolling response, Bioconjug. Chem. 19 (11) (2008) 2105-2109.

[315] M.T. Stephan, D.J. Irvine, Enhancing cell therapies from the outside in: cell surface engineering using synthetic nanomaterials, Nano Today 6 (3) (2011) 309-325.

[316] M.R. Choi, et al., A cellular Trojan Horse for delivery of therapeutic nanoparticles into tumors, Nano Lett. 7 (12) (2007) 3759-3765.

[317] R.J. Melder, C.A. Kristensen, L.L. Munn, R.K. Jain, Modulation of A-NK cell rigidity: in vitro characterization and in vivo implications for cell delivery, Biorheology 38 (2-3) (2001) 151-159.

[318] C. Perez-Campana, et al., Biodistribution of different sized nanoparticles assessed by positron emission tomography: a general strategy for direct activation of metal oxide particles, ACS Nano 7 (4) (2013) 3498-3505.

[319] N. Hoshyar, S. Gray, H. Han, G. Bao, The effect of nanoparticle size on in vivo pharmacokinetics and cellular interaction, Nanomedicine (London, England) 11 (6) (2016) 673-692.

[320] C. Zhang, et al., Labeling stem cells with a near-infrared fluorescent heptamethine dye for noninvasive optical tracking, Cell Transplant. 20 (5) (2011) 741-751.

[321] J. Gao, J.E. Dennis, R.F. Muzic, M. Lundberg, A.I. Caplan, The dynamic in vivo distribution of bone marrow-derived mesenchymal stem cells after infusion, Cells Tissues Organs 169 (1) (2001) 12-20.

[322] Y.J. Choi, et al., Visualization of the biological behavior of tumor-associated macrophages in living mice with colon cancer using multimodal optical reporter gene imaging, Neoplasia (New York, N.Y.) 18 (3) (2016) 133-141.

[323] J. Leibacher, R. Henschler, Biodistribution, migration and homing of systemically applied mesenchymal stem/stromal cells, Stem Cell Res Ther 7 (1) (2016) 7.

[324] D. Ritchie, et al., In vivo tracking of macrophage activated killer cells to sites of metastatic ovarian carcinoma, Cancer Immunol. Immunother. 56 (2) (2007) $155-163$.

[325] C.A. Boswell, et al., Comparative physiology of mice and rats: radiometric measurement of vascular parameters in rodent tissues, Mol. Pharm. 11 (5) (2014) $1591-1598$.

[326] M. Eltze, Functional evidence for an alpha 1B-adrenoceptor mediating contraction of the mouse spleen, Eur. J. Pharmacol. 311 (2-3) (1996) 187-198.

[327] R.J. Shephard, Responses of the human spleen to exercise, J. Sports Sci. 34 (10) (2016) 929-936.

[328] R.J. Melder, et al., Systemic distribution and tumor localization of adoptively transferred lymphocytes in mice: comparison with physiologically based pharmacokinetic model, Neoplasia (New York, N.Y.) 4 (1) (2002) 3-8.

[329] F. Combes, A.M. Sofias, Mononuclear but Not Polymorphonuclear Phagocyte Depletion Increases Circulation Times and Improves Mammary Tumor-homing Efficiency of Donor Bone Marrow-derived Monocytes, 11(11) (2019).

[330] S.P. Davies, G.M. Reynolds, Z. Stamataki, Clearance of apoptotic cells by tissue epithelia: a putative role for hepatocytes in liver efferocytosis, Front. Immunol. 9 (2018) 44.

[331] Bosman GJCGM, Survival of red blood cells after transfusion: processes and consequences, Front. Physiol. 4 (2013) 376.

[332] K.S. Ravichandran, Beginnings of a good apoptotic meal: the find-me and eat-me signaling pathways, Immunity 35 (4) (2011) 445-455. 
[333] S. Krahling, M.K. Callahan, P. Williamson, R.A. Schlegel, Exposure of phosphati dylserine is a general feature in the phagocytosis of apoptotic lymphocytes by macrophages, Cell Death Differ. 6 (2) (1999) 183-189.

[334] D.E. Discher, How does CD47-SIRPa 'don't eat me signal' physically signal self, Blood 122 (21) (2013) 953

[335] E.M. Carrington, et al., Anti-apoptotic proteins BCL-2, MCL-1 and A1 summate collectively to maintain survival of immune cell populations both in vitro and in vivo, Cell Death Differ. 24 (2017) 878.

[336] A. Kurtz, Mesenchymal stem cell delivery routes and fate, Int. J. Stem Cells 1 (1) (2008) 1-7.

[337] E. Kolaczkowska, P. Kubes, Neutrophil recruitment and function in health and inflammation, Nat. Rev. Immunol. 13 (3) (2013) 159-175.

[338] Y. Ueda, D.W. Cain, M. Kuraoka, M. Kondo, G. Kelsoe, IL-1R type I-dependent hemopoietic stem cell proliferation is necessary for inflammatory granulopoiesis and reactive neutrophilia, J. Immunol. (Baltimore, Md. : 1950) 182 (10) (2009) 6477-6484.

[339] J.J. Cream, Prednisolone-induced granulocytosis, Br. J. Haematol. 15 (3) (1968) 259-268.

[340] Y. Shoenfeld, Y. Gurewich, L.A. Gallant, J. Pinkhas, Prednisone-induced leukocytosis. Influence of dosage, method and duration of administration on the degree of leukocytosis, Am. J. Med. 71 (5) (1981) 773-778.

[341] M. Nakagawa, et al., Glucocorticoid-induced granulocytosis: contribution of marrow release and demargination of intravascular granulocytes, Circulation 98 (21) (1998) 2307-2313.

[342] D.M.H. Cavalcanti, et al., Endogenous glucocorticoids control neutrophil mobilization from bone marrow to blood and tissues in non-inflammatory conditions, $\mathrm{Br}$. J. Pharmacol. 152 (8) (2007) 1291-1300.
[343] S. Ronchetti, E. Ricci, G. Migliorati, M. Gentili, C. Riccardi, How glucocorticoids affect the neutrophil life, Int. J. Mol. Sci. 19 (12) (2018) 4090.

[344] G. von Maltzahn, et al., Nanoparticles that communicate in vivo to amplify tumour targeting, Nat. Mater. 10 (7) (2011) 545-552.

[345] A.M. Sofias, T. Andreassen, S. Hak, Nanoparticle ligand-decoration procedures affect in vivo interactions with immune cells, Mol. Pharm. 15 (12) (2018) 5754-5761.

[346] A.N. Miliotou, L.C. Papadopoulou, CAR T-cell therapy: a new era in cancer im munotherapy, Curr. Pharm. Biotechnol. 19 (1) (2018) 5-18.

[347] C. Fountzilas, S. Patel, D. Mahalingam, Review: oncolytic virotherapy, updates and future directions, Oncotarget 8 (60) (2017) 102617-102639.

[348] I.C. Miller, M. Gamboa Castro, J. Maenza, J.P. Weis, G.A. Kwong, Remote contro of mammalian cells with heat-triggered gene switches and photothermal pulse trains, ACS Synth. Biol. 7 (4) (2018) 1167-1173.

[349] S. Goverdhana, et al., Regulatable gene expression systems for gene therapy applications: progress and future challenges, Mol. Ther. 12 (2) (2005) 189-211.

[350] G. Zhong, H. Wang, C.C. Bailey, G. Gao, M. Farzan, Rational design of aptazyme riboswitches for efficient control of gene expression in mammalian cells, eLife 5 (2016) e18858.

[351] B.D. Brown, M.A. Venneri, A. Zingale, L. Sergi Sergi, L. Naldini, Endogenous microRNA regulation suppresses transgene expression in hematopoietic lineages and enables stable gene transfer, Nat. Med. 12 (5) (2006) 585-591.

[352] A. Aalipour, et al., Engineered immune cells as highly sensitive cancer diagnostics, Nat. Biotechnol. 37 (2019) 531-539.

[353] M. Rath, I. Muller, P. Kropf, E.I. Closs, M. Munder, Metabolism via arginase or nitric oxide synthase: two competing arginine pathways in macrophages, Front Immunol. 5 (2014) 532. 